\title{
NUMERICAL VARIATIONAL METHODS APPLIED TO CYLINDER BUCKLING
}

\author{
JIŘÍ HORÁK*, GABRIEL J. LORD ${ }^{\dagger}$, AND MARK A. PELETIER
}

\begin{abstract}
We review and compare different computational variational methods applied to a system of fourth order equations that arises as a model of cylinder buckling. We describe both the discretization and implementation, in particular how to deal with a 1 dimensional null space. We show that we can construct many different solutions from a complex energy surface. We examine numerically convergence in the spatial discretization and in the domain size. Finally we give a physical interpretation of some of the solutions found.
\end{abstract}

1. Introduction. We describe complementary approaches to finding solutions of systems of fourth order elliptic PDEs. The techniques are applied to a problem that arises in the classic treatment of an isotropic cylindrical shell under axial compression but are also applicable to a wide range of problems such as waves on a suspension bridge $[4,6]$, the Fučík spectrum of the Laplacian [7], or the formation of microstructure [12,3].

The cylindrical shell offers a computationally challenging and physically relevant problem with a complex energy surface. We take as our model for the shell the Von Kármán-Donnell equations which can be rescaled [5] to the form

$$
\begin{aligned}
& \Delta^{2} w+\lambda w_{x x}-\phi_{x x}-2[w, \phi]=0 \\
& \Delta^{2} \phi+w_{x x}+[w, w]=0
\end{aligned}
$$

where the bracket is defined as

$$
[u, v]=\frac{1}{2} u_{x x} v_{y y}+\frac{1}{2} u_{y y} v_{x x}-u_{x y} v_{x y} .
$$

The function $w$ is a scaled inward radial displacement measured from the unbuckled (fundamental) state, $\phi$ is the Airy stress function, and $\lambda \in(0,2)$ is a load parameter. The unknowns $w$ and $\phi$ are defined on a two-dimensional spatial domain $\Omega=(-a, a) \times(-b, b)$, where $x \in(-a, a)$ is the axial and $y \in(-b, b)$ is the tangential coordinate. Since the $y$-domain $(-b, b)$ represents the circumference of the cylinder, the following boundary conditions are prescribed:

$$
\begin{aligned}
& w \text { is periodic in } y, \text { and } w_{x}=(\Delta w)_{x}=0 \text { at } x= \pm a, \\
& \phi \text { is periodic in } y, \text { and } \phi_{x}=(\Delta \phi)_{x}=0 \text { at } x= \pm a,
\end{aligned}
$$

as shown in Fig. 1.1 (i), (ii).

1.1. Functional setting. We search for weak solutions $w, \phi$ of $(1.1-1.4)$ in the space

$$
X=\left\{\psi \in H^{2}(\Omega): \psi_{x}( \pm a, \cdot)=0, \psi \text { is periodic in } y, \text { and } \int_{\Omega} \psi=0\right\}
$$

with norm

$$
\|w\|_{X}^{2}=\int_{\Omega}\left(\Delta w^{2}+\Delta \phi_{1}^{2}\right)
$$

where $\phi_{1} \in H^{2}(\Omega)$ is the unique solution of

$$
\Delta^{2} \phi_{1}=-w_{x x}, \quad \phi_{1} \text { satisfies }(1.4 \mathrm{~b}), \quad \text { and } \quad \int_{\Omega} \phi_{1}=0 .
$$

\footnotetext{
*Universität Köln, Germany

${ }^{\dagger}$ Heriot-Watt University, Edinburgh, United Kingdom

${ }_{\ddagger}$ Technische Universiteit Eindhoven, The Netherlands
} 


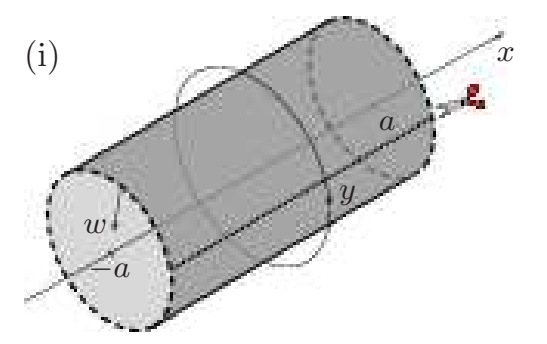

periodic boundary condition for $w, \phi$

$w_{\nu}=(\Delta w)_{\nu}=\phi_{\nu}=(\Delta \phi)_{\nu}=0$
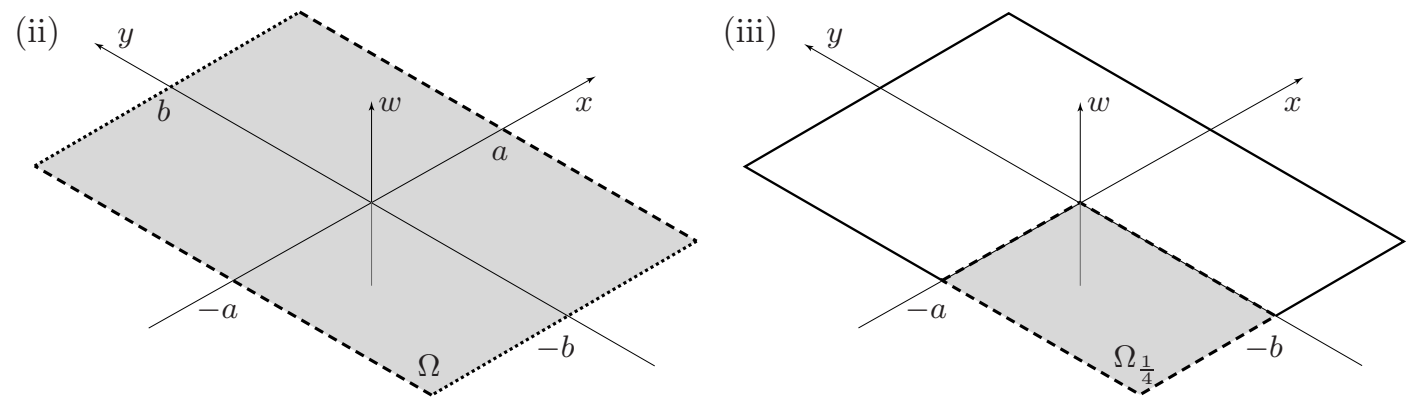

FIG. 1.1. (i) The geometry of the cylinder, (ii) the computational domain and the boundary conditions, (iii) one quarter of the domain and the corresponding boundary conditions.

This norm is equivalent to the $H^{2}$-norm on $X$, and with the appropriate inner product $\langle\cdot, \cdot\rangle_{X}$ the space $X$ is a Hilbert space. Alternatively, if the load parameter $\lambda \in(0,2)$ is fixed, another norm

$$
\|w\|_{X, \lambda}^{2}=\int_{\Omega}\left(\Delta w^{2}+\Delta \phi_{1}^{2}-\lambda w_{x}^{2}\right)
$$

can be used. Because of the estimate

$$
\int_{\Omega} w_{x}^{2}=-\int_{\Omega} w w_{x x}=\int_{\Omega} w \Delta^{2} \phi_{1}=\int_{\Omega} \Delta w \Delta \phi_{1} \leq \frac{1}{2} \int_{\Omega} \Delta w^{2}+\frac{1}{2} \int_{\Omega} \Delta \phi_{1}^{2}=\frac{1}{2}\|w\|_{X}^{2},
$$

it is equivalent to $\|\cdot\|_{X}$ and hence also to the $H^{2}$-norm on $X$. The corresponding inner product will be denoted $\langle\cdot, \cdot\rangle_{X, \lambda}$.

Equations (1.1-1.2) are related to the stored energy $E$, the average axial shortening $S$, and the total potential given by

$$
E(w):=\frac{1}{2} \int_{\Omega}\left(\Delta w^{2}+\Delta \phi^{2}\right), \quad S(w):=\frac{1}{2} \int_{\Omega} w_{x}^{2}, \quad F_{\lambda}=E-\lambda S .
$$

Note that the function $\phi$ in (1.6) is determined from $w$ by solving (1.2) with boundary conditions (1.4b). All the functionals $E, S$, and $F_{\lambda}$ belong to $C^{1}(X)$, i.e., are continuously Fréchet differentiable.

The fact that (1.1) is a reformulation of the stationarity condition $F_{\lambda}^{\prime}=E^{\prime}-\lambda S^{\prime}=0$ will be important in Sec. 2, and we therefore briefly sketch the argument. It is easy to see that

$$
S^{\prime}(w) \cdot h=-\int_{\Omega} w_{x x} h .
$$

For $E^{\prime}(w) \cdot h$, let $w, \phi \in X$ solve $(1.2)$ and $h, \psi \in X$ solve $\Delta^{2} \psi=-h_{x x}-[h, h]-2[w, h]$. Then, assuming sufficient regularity on $w$,

$$
\begin{aligned}
E(w+h)-E(w) & =\int_{\Omega} \Delta w \Delta h+\frac{1}{2} \int_{\Omega}(\Delta h)^{2}+\int_{\Omega} \Delta \phi \Delta \psi+\frac{1}{2} \int_{\Omega}(\Delta \psi)^{2} \\
& =\int_{\Omega}\left(h \Delta^{2} w-h \phi_{x x}-2[w, h] \phi\right)+\frac{1}{2} \int_{\Omega}(\Delta h)^{2}+\frac{1}{2} \int_{\Omega}(\Delta \psi)^{2}-\int_{\Omega}[h, h] \phi,
\end{aligned}
$$


where we used integration by parts several times. The last three integrals are $O\left(\|h\|_{X}^{2}\right)$ for $\|h\|_{X} \rightarrow$ 0 and it can be shown by integration by parts that

$$
\int_{\Omega}[w, h] \phi=\int_{\Omega} h[w, \phi] .
$$

Therefore

$$
F_{\lambda}^{\prime}(w) \cdot h=E^{\prime}(w) \cdot h-\lambda S^{\prime}(w) \cdot h=\int_{\Omega} h\left(\Delta^{2} w+\lambda w_{x x}-\phi_{x x}-2[w, \phi]\right) .
$$

1.2. Review of some variational numerical methods. We now describe the variational methods used to find numerical approximations of critical points of the total potential $F_{\lambda}$. In our numerical experiments these methods are accompanied by Newton's method and continuation. The advantage of this approach is that it combines the knowledge of global features of the energy landscape with local ones of a neighborhood of a critical point. The details related to spatial discretization will be discussed in Sec. 2, the Newton-based methods in Sec. 3.

1.2.1. Steepest descent method (SDM). Let the load parameter $\lambda \in(0,2)$ be fixed; we work in a discretized version of $\left(X,\langle\cdot, \cdot\rangle_{X, \lambda}\right)$. We try to minimize the total potential $F_{\lambda}$ by following its gradient flow. We solve the initial value problem

$$
\frac{d}{d t} w(t)=-\nabla_{\lambda} F_{\lambda}(w(t)), \quad w(0)=w_{0},
$$

with a suitable starting point $w_{0}$ on some interval $(0, T]$. This problem is then discretized in $t$.

In [5] it was shown that $w=0$ is a local minimizer of $F_{\lambda}$. Indeed, if $\left\|w_{0}\right\|_{X, \lambda}$ is small, the numerical solution $w(t)$ converges to zero as $t$ tends to infinity. If, on the other hand, $\left\|w_{0}\right\|_{X, \lambda}$ is large, the numerical solution $w(t)$ stays bounded away from zero. In most of our experiments, the numerical algorithm did not converge for $t \rightarrow \infty$ in the large norm case. The only exception for a relatively small value of $\lambda$ will be mentioned later in Sec. 5.3. Nevertheless, for a sufficiently large computational domain $\Omega$ and a sufficiently large $t>0$ we obtain $F_{\lambda}(w(t))<0$. Such a state $w(t)$ is needed for the mountain pass algorithm as explained below. Existence of this state was also proved in [5].

1.2.2. Mountain-pass algorithm (MPA). The algorithm was first proposed in [2] for a second order elliptic problem in $1 \mathrm{D}$ and extended in [6] to a fourth-order problem in $2 \mathrm{D}$. We give a brief description of the algorithm here.

Let the load $\lambda \in(0,2)$ be fixed; we work again in a discretized version of $\left(X,\langle\cdot, \cdot\rangle_{X, \lambda}\right)$. We denote $w_{1}=0$ the local minimum of $F_{\lambda}$ and take a point $w_{2}$ such that $F_{\lambda}\left(w_{2}\right)<0$ (in practice this point is found using the SDM). We take a discretized path $\left\{z_{m}\right\}_{m=0}^{p}$ connecting $z_{0}=w_{1}$ with $z_{p}=w_{2}$. After finding the point $z_{m}$ at which $F_{\lambda}$ is maximal along the path, this point is moved a small distance in the direction of the steepest descent $-\nabla_{\lambda} F_{\lambda}\left(z_{m}\right)$. Thus the path has been deformed and the maximum of $F_{\lambda}$ lowered. This deforming of the path is repeated until the maximum along the path cannot be lowered any more: a critical point $w_{\mathrm{MP}}$ has been reached. Figure 1.2 illustrates the main idea of the method.

The mountain-pass algorithm is local in its nature. The numerical solution $w_{\mathrm{MP}}$ it finds has the mountain-pass property in a certain neighborhood only. The choice of the path endpoint $w_{2}$ may influence to which critical point the algorithm converges. Different choices of $w_{2}$ are in turn achieved by choosing different initial points $w_{0}$ in the SDM.

1.2.3. Constrained steepest descent method (CSDM). We fix the amount of shortening $S$ of the cylinder. This is often considered as what actually occurs in experiments. We work now in a discretized version of $\left(X,\langle\cdot, \cdot\rangle_{X}\right)$. Let $C>0$ be a fixed number and define a set of $w$ with constant shortening

$$
\mathscr{M}=\{w \in X: S(w)=C\} .
$$




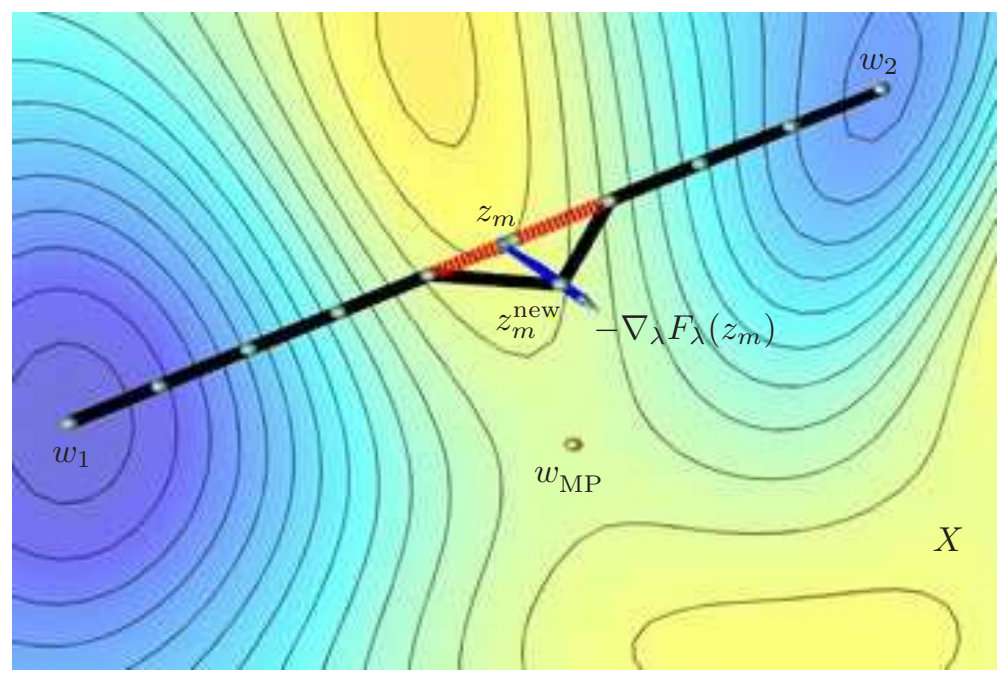

FIG. 1.2. Deforming the path in the main loop of the mountain pass algorithm: point $z_{m}$ is moved a small distance in the direction $-\nabla_{\lambda} F_{\lambda}\left(z_{m}\right)$ and becomes $z_{m}^{\text {new }}$. This step is repeated until the mountain pass point $w_{\mathrm{MP}}$ is reached.

Critical points of $E$ under this constraint are critical points of $F_{\lambda}$, where $\lambda$ is a Lagrange multiplier. The simplest such points are local minima of the stored energy $E$ on $\mathscr{M}$. We need to follow the gradient flow of $E$ on $\mathscr{M}$, hence we solve the initial value problem

$$
\frac{d}{d t} w(t)=-P_{w(t)} \nabla E(w(t)), \quad w(0)=w_{0} \in \mathscr{M},
$$

for $t>0 . P_{w}$ denotes the orthogonal projection in $X$ on the tangent space of $\mathscr{M}$ at $w \in \mathscr{M}$ :

$$
P_{w} u=u-\frac{\langle\nabla S(w), u\rangle_{X}}{\|\nabla S(w)\|_{X}^{2}} \nabla S(w) .
$$

The details of the algorithm can be found in [4]. The initial value problem is solved by repeating the following two steps: given a point $w \in \mathscr{M}$ find $\bar{w}=w-\Delta t P_{w} \nabla E(w)$, where $\Delta t>0$ is small, and define $w_{\text {new }}=c \bar{w}$, where the scaling coefficient $c$ is chosen so that $w_{\text {new }} \in \mathscr{M}$. The algorithm is stopped when $\left\|P_{w} \nabla E(w)\right\|_{X}$ is smaller than a prescribed tolerance. The corresponding load is given by

$$
\lambda=\frac{\langle\nabla S(w), \nabla E(w)\rangle_{X}}{\|\nabla S(w)\|_{X}^{2}}
$$

1.2.4. Constrained mountain-pass algorithm (CMPA). Let $C>0$ and $\mathscr{M}$ be the set of $w$ with constant shortening given in (1.8). We would like to find mountain-pass points of $E$ on $\mathscr{M}$. The method has been described in [4] in detail. We need two local minima $w_{1}, w_{2}$ of $E$ on $\mathscr{M}$ which can be found using the CSDM. The algorithm is then similar to the MPA. We take a discretized path $\left\{z_{m}\right\}_{m=0}^{p} \subset \mathscr{M}$ connecting $z_{0}=w_{1}$ with $z_{p}=w_{2}$. After finding the point $z_{m}$ at which $E$ is maximal along the path, this point is moved a small distance in the tangent space to $\mathscr{M}$ at $z_{m}$ in the direction of the steepest descent $-P_{z_{m}} \nabla E\left(z_{m}\right)$ and than scaled (as in the CSDM) to come back to $\mathscr{M}$. Thus the path has been deformed on $\mathscr{M}$ and the maximum of $E$ lowered. This deforming of the path is repeated until the maximum along the path cannot be lowered any more: a mountain-pass point of $E$ on $\mathscr{M}$ has been reached. The load $\lambda$ is computed as in the CSDM.

The choice of the end points $w_{1}$ and $w_{2}$ will in general influence to which critical point the algorithm converges. 
1.3. Computational Domains. We consider the problem on the domain $\Omega$ (Figure 1.1 (ii)) both without further restraints and under a symmetry assumption, which reduces the computational complexity. In the latter case we assume

$$
\begin{aligned}
w(x, y) & =w(-x, y)=w(x,-y) \\
\phi(x, y) & =\phi(-x, y)=\phi(x,-y)
\end{aligned} \quad \text { for }(x, y) \in \Omega .
$$

By looking for solutions $w, \phi \in X$ that satisfy (1.9) the domain is effectively reduced to one quarter: $\Omega_{\frac{1}{4}}=(-a, 0) \times(-b, 0)$ as shown in Figure 1.1 (iii). One needs to solve (1.1-1.2) only on $\Omega_{\frac{1}{4}}$ with the boundary conditions

$$
w_{\nu}=(\Delta w)_{\nu}=\phi_{\nu}=(\Delta \phi)_{\nu}=0 \quad \text { on } \partial \Omega_{\frac{1}{4}},
$$

where $\nu$ denotes the outward normal direction to the boundary. Hence we search for weak solutions of $(1.1-1.2),(1.10)$ in the space

$$
X_{\frac{1}{4}}=\left\{\psi \in H^{2}\left(\Omega_{\frac{1}{4}}\right): \psi_{\nu}=0 \text { on } \partial \Omega_{\frac{1}{4}} \text {, and } \int_{\Omega_{\frac{1}{4}}} \psi=0\right\} .
$$

We can then use (1.9) to extend these functions to the whole $\Omega$.

We have performed numerical experiments both with and without the symmetry assumption. For the sake of simplicity we will give a detailed description of the numerical methods for the second case only where the boundary conditions are the same on all sides of $\Omega_{\frac{1}{4}}$. The first case with periodic conditions on two sides of $\Omega$ is very similar and will be briefly mentioned in Remark 2.1.

1.4. Solving the biharmonic equation. In order to obtain $\phi$ for a given $w$, one has to solve (1.2); to compute the norm of $w$, one has to solve (1.5). Both problems are of the form

$$
\Delta^{2} \psi=f \text { in } \Omega_{\frac{1}{4}}, \quad \psi_{\nu}=(\Delta \psi)_{\nu}=0 \text { on } \partial \Omega_{\frac{1}{4}}, \quad \int_{\Omega_{\frac{1}{4}}} \psi=0,
$$

where $f \in L^{1}\left(\Omega_{\frac{1}{4}}\right)$ is given. If $\int_{\Omega_{\frac{1}{4}}} f=0$, then (1.11) has a unique weak solution $\psi$ in $X_{\frac{1}{4}}$. It is a straightforward calculation to verify that the right-hand sides of equations in (1.2) and (1.5) have zero average.

In the discretization described below the problem (1.11) is treated as a system:

$$
\begin{aligned}
& -\Delta u=f \\
& -\Delta v=u
\end{aligned} \quad \text { in } \Omega_{\frac{1}{4}}, \quad u_{\nu}=v_{\nu}=0 \text { on } \partial \Omega_{\frac{1}{4}}, \quad \int_{\Omega_{\frac{1}{4}}} u=\int_{\Omega_{\frac{1}{4}}} v=0 .
$$

The system has a unique weak solution $(u, v) \in\left(H^{1}\left(\Omega_{\frac{1}{4}}\right)\right)^{2}$. Since the domain $\Omega_{\frac{1}{4}}$ has no reentrant corners, Theorem 1.4.5 of [9] guarantees that $v \in H^{2}\left(\Omega_{\frac{1}{4}}\right)$ and therefore that the two formulations are equivalent.

2. Finite difference discretization. We discretize the domain $\Omega_{\frac{1}{4}}$ by a uniform mesh $\left(x_{m}, y_{n}\right) \in \Omega_{\frac{1}{4}}$ with $M$ points in the $x$-direction and $N$ points in the $y$-direction:

$$
\begin{aligned}
x_{m} & =-a+\left(m-\frac{1}{2}\right) \Delta x, & m & \in\{1 \ldots, M\}, \\
y_{n} & =-b+\left(n-\frac{1}{2}\right) \Delta y, & n & \in\{1 \ldots, N\},
\end{aligned}
$$

where $\Delta x=a / M, \Delta y=b / N$. We represent the values of some function $w$ on $\Omega_{\frac{1}{4}}$ at these points by a vector $w=\left(w_{i}\right)_{i=1}^{M N}$, where $w_{i}=w\left(x_{m}, y_{n}\right)$ and $i=(n-1) M+m$. In our notation we will not distinguish between $w$ as a function and $w$ as a corresponding vector. The vector $w$ can also be interpreted as a block vector with $N$ blocks, each containing $M$ values of a single row of the mesh. We introduce the following conventions for notation: 
- For two matrices $A^{M}=\left(a_{i j}\right)_{i, j=1}^{M}, B^{N}=\left(b_{k \ell}\right)_{k, \ell=1}^{N}$ we define $A^{M} \otimes B^{N}:=\left(b_{k \ell} A^{M}\right)_{k, \ell=1}^{N}$, which is an $N \times N$ block matrix, each block is an $M \times M$ matrix.

- For two vectors $u=\left(u_{i}\right)_{i=1}^{M N}, v=\left(v_{i}\right)_{i=1}^{M N}$ we define $u \odot v=\left(u_{i} v_{i}\right)_{i=1}^{M N}$, i.e., a product of the components.

To discretize second derivatives we use the standard central finite differences (with Neumann boundary conditions [10]). Let $I d^{M}$ denote the $M \times M$ identity matrix and define another $M \times M$ matrix

$$
A_{2}^{M}=\left[\begin{array}{rrrrr}
1 & -1 & & & \\
-1 & 2 & -1 & & \\
& \ddots & \ddots & \ddots & \\
& & -1 & 2 & -1 \\
& & & -1 & 1
\end{array}\right]
$$

The second derivatives $-\partial_{x x},-\partial_{y y}$ and the biharmonic operator $\Delta^{2}$ with the appropriate boundary conditions are approximated by

$$
\boldsymbol{A}_{x x}=\frac{1}{\Delta x^{2}} A_{2}^{M} \otimes I d^{N}, \quad \boldsymbol{A}_{y y}=\frac{1}{\Delta y^{2}} I d^{M} \otimes A_{2}^{N}, \quad \boldsymbol{A}_{\Delta^{2}}=\left(\boldsymbol{A}_{x x}+\boldsymbol{A}_{y y}\right)^{2},
$$

respectively.

2.1. Discretization of $E, S$, and the bracket $[\cdot, \cdot]$. Supposing that we can solve the discretized version of (1.2)

$$
\boldsymbol{A}_{\Delta^{2}} \phi-\boldsymbol{A}_{x x} w+[w, w]_{2}=\mathbf{0},
$$

we can also evaluate the energy $E$ and the shortening $S$ :

$$
E(w)=2\left(w^{T} \boldsymbol{A}_{\Delta^{2}} w+\phi^{T} \boldsymbol{A}_{\Delta^{2}} \phi\right) \Delta x \Delta y, \quad S(w)=2\left(w^{T} \boldsymbol{A}_{x x} w\right) \Delta x \Delta y .
$$

In order to solve (2.1) we need to be able to solve the biharmonic equation and we need to choose a discretization of the bracket $[\cdot, \cdot]$. This bracket appears in the equations in two different roles: in equation (1.2) the bracket is part of the mapping $w \mapsto \phi$, and therefore of the definition of the energy $E$; in equation (1.1), which represents the stationarity condition $E^{\prime}-\lambda S^{\prime}=0$, the bracket appears as a result of differentiating $E$ with respect to $w$ and applying partial integration. As a result, we need to use two different forms of discretization for the two cases.

In both cases the bracket requires a discretization of the mixed derivative $\partial_{x y}$. One choice is to use one-sided finite differences. Define $M \times M$ matrices

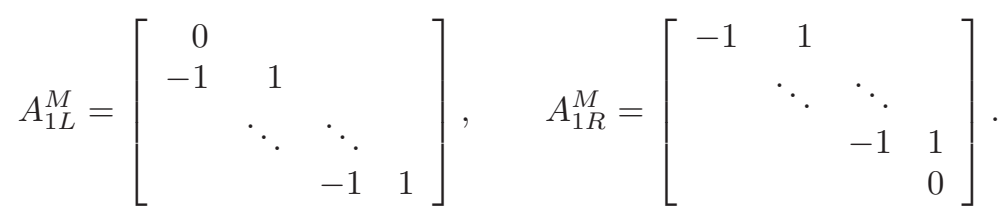

We choose either left or right-sided differences represented by these matrices, respectively, let $A_{1}^{M}$ denote our choice (cf. Sec. 5.1). The derivatives $\partial_{x}, \partial_{y}$, and $-\partial_{x y}$ are approximated by

$$
\boldsymbol{A}_{x}=\frac{1}{\Delta x} A_{1}^{M} \otimes I d^{N}, \quad \boldsymbol{A}_{y}=\frac{1}{\Delta y} I d^{M} \otimes A_{1}^{N}, \quad \boldsymbol{A}_{x y}=-\boldsymbol{A}_{x} \boldsymbol{A}_{y} .
$$

For the definition of $\phi$ in terms of $w$ (equation (1.2)) we choose

$$
[w, w]_{2}=\left(\boldsymbol{A}_{x x} w\right) \odot\left(\boldsymbol{A}_{y y} w\right)-\left(\boldsymbol{A}_{x y} w\right) \odot\left(\boldsymbol{A}_{x y} w\right),
$$

and the corresponding choice for equation (1.1) is

$$
[w, \phi]_{1}=\frac{1}{2} \boldsymbol{A}_{y y}\left\{\left(\boldsymbol{A}_{x x} w\right) \odot \phi\right\}+\frac{1}{2} \boldsymbol{A}_{x x}\left\{\left(\boldsymbol{A}_{y y} w\right) \odot \phi\right\}-\boldsymbol{A}_{x y}^{T}\left\{\left(\boldsymbol{A}_{x y} w\right) \odot \phi\right\} .
$$

These two definitions are related in the sense given in (1.7): $[w, h]_{2}^{T} \phi=h^{T}[w, \phi]_{1}$ for all $h$.

With these definitions the partial derivatives of discretized $E$ and $S$ with respect to the components of $w$ are given by

$$
E^{\prime}(w)=\boldsymbol{A}_{\Delta^{2}} w+\boldsymbol{A}_{x x} \phi-2[w, \phi]_{1}, \quad S^{\prime}(w)=\boldsymbol{A}_{x x} w .
$$


2.2. Solving the discretized biharmonic equation. Matrix $\boldsymbol{A}_{\Delta^{2}}$ is symmetric and has a one-dimensional nullspace: $\boldsymbol{A}_{\Delta^{2}} \mathbf{1}=\mathbf{0}$, where $\mathbf{1}$ and $\mathbf{0}$ are vectors with $M N$-components which are all one and all zero, respectively. The same is true for $\boldsymbol{A}_{x x}$ and $\boldsymbol{A}_{y y}$. For a given vector $f$ we would like to solve

$$
\boldsymbol{A}_{\Delta^{2}} \psi=f, \quad \mathbf{1}^{T} \psi=0 .
$$

A unique solution exists if and only if $f$ has zero average, i.e., $\mathbf{1}^{T} f=0$. So we must verify that the discretized versions of the right-hand sides in (1.2), (1.5) satisfy this condition. Let $w \in \mathbb{R}^{M N}$, then

$$
\begin{aligned}
\mathbf{1}^{T} \boldsymbol{A}_{x x} w & =0, \\
\mathbf{1}^{T}[w, w]_{2} & =\left(\boldsymbol{A}_{x x} w\right)^{T}\left(\boldsymbol{A}_{y y} w\right)-\left(\boldsymbol{A}_{x y} w\right)^{T}\left(\boldsymbol{A}_{x y} w\right)=w^{T} \boldsymbol{A}_{x x} \boldsymbol{A}_{y y} w-w^{T} \boldsymbol{A}_{x y}^{T} \boldsymbol{A}_{x y} w=0,
\end{aligned}
$$

where the last equality holds because $\boldsymbol{A}_{x}^{T} \boldsymbol{A}_{x}=\boldsymbol{A}_{x x}$ and $\boldsymbol{A}_{y}^{T} \boldsymbol{A}_{y}=\boldsymbol{A}_{y y}$, and because the $x$ - and $y$-matrices commute. We have, in fact, shown that the integration by parts formula from the continuous case holds for our choice of spatial discretization. This is not true for an arbitrary discretization but is key for a successful scheme.

The inverse of matrix $\boldsymbol{A}_{\Delta^{2}}$ on the subspace of vectors with zero average, denoted with a slight abuse of notation by $\boldsymbol{A}_{\Delta^{2}}^{-1}$, can be found, for example, using the fast cosine transform described below in Sec. 2.4.

2.3. Computing the gradient. The variational methods of this paper are based on a steepest descent flow and modifications of this algorithm. The direction of the steepest descent of $E$ at a point $w \in X$ is opposite to the gradient of $E$ at $w$. The gradient is the Riesz representative of the Fréchet derivative and hence we need to find a vector $u \in X$, such that $E^{\prime}(w) \cdot v=\langle u, v\rangle$ for all $v \in X$. The inner product is either $\langle\cdot, \cdot\rangle_{X}$ or $\langle\cdot, \cdot\rangle_{X, \lambda}$ and hence the gradient depends on the choice of the inner product. We use the notation $u=\nabla E(w)$ for the gradient in $\left(X,\langle\cdot, \cdot\rangle_{X}\right)$ and $u=\nabla_{\lambda} E(w)$ for the gradient in $\left(X,\langle\cdot, \cdot\rangle_{X, \lambda}\right)$. To find the discretized version of the gradient, we first need to discretize the inner product.

Let $u, v \in \mathbb{R}^{M N}, \mathbf{1}^{T} u=\mathbf{1}^{T} v=0$. The inner product is evaluated in the following way:

$$
\begin{aligned}
\langle u, v\rangle_{X, \lambda} & =4\left(u^{T} \boldsymbol{A}_{\Delta^{2}} v+\phi_{1}^{u T} \boldsymbol{A}_{\Delta^{2}} \phi_{1}^{v}-\lambda u^{T} \boldsymbol{A}_{x x} v\right) \Delta x \Delta y \\
& =4\left(u^{T}\left(\boldsymbol{A}_{\Delta^{2}}+\boldsymbol{A}_{x x} \boldsymbol{A}_{\Delta^{2}}^{-1} \boldsymbol{A}_{x x}-\lambda \boldsymbol{A}_{x x}\right) v\right) \Delta x \Delta y,
\end{aligned}
$$

where $\phi_{1}^{u}, \phi_{1}^{v}$ are solutions of the discretized version of (1.5) with $w$ replaced by $u$ and $v$, respectively, and we assume that we work on $\Omega_{\frac{1}{4}}$. For $w \in \mathbb{R}^{M N}, \mathbf{1}^{T} w=0$ the Riesz representative of $E^{\prime}(w)$ given in (2.7) is computed as

$$
\nabla_{\lambda} E(w)=\left(\boldsymbol{A}_{\Delta^{2}}+\boldsymbol{A}_{x x} \boldsymbol{A}_{\Delta^{2}}^{-1} \boldsymbol{A}_{x x}-\lambda \boldsymbol{A}_{x x}\right)^{-1} E^{\prime}(w) .
$$

As in the case of $\boldsymbol{A}_{\Delta^{2}}^{-1}$ we abused notation here since the inverse only makes sense on a subspace of vectors with zero average. It can be easily verified that $\mathbf{1}^{T} E^{\prime}(w)=0$. The numerical evaluation of $\nabla_{\lambda} S$ and of the $\langle\cdot, \cdot\rangle_{X}$-gradients is similar.

2.4. Fourier coordinates. In Fourier coordinates most of the finite difference operators become diagonal matrices. This increases the efficiency of the numerical algorithm and makes it possible to easily find the inverse of matrices like $\boldsymbol{A}_{\Delta^{2}}$. See for example [1].

On a uniform grid, it is a standard procedure to apply some form of the fast Fourier transform to diagonalize finite difference matrices like $A_{2}^{M}$ (see, e.g., [11]). Due to the Neumann boundary conditions (1.10) we need to employ the fast cosine transform. We define $M \times M$ matrices

$$
C_{\mathrm{f}}^{M}=\frac{1}{\sqrt{2 M}}\left(2 \cos \frac{(i-1)(2 j-1) \pi}{2 M}\right)_{i, j=1}^{M}, \quad C_{\mathrm{b}}^{M}=\frac{1}{\sqrt{2 M}}\left[\begin{array}{c|l}
1 & \left(2 \cos \frac{(2 i-1)(j-1) \pi}{2 M}\right)_{i=1, j=2}^{M, M} \\
\vdots &
\end{array}\right],
$$


which have the following properties:

$$
C_{\mathrm{f}}^{M} C_{\mathrm{b}}^{M}=I d^{M}, \quad C_{\mathrm{f}}^{M} A_{2}^{M} C_{\mathrm{b}}^{M}=\Lambda^{M},
$$

where $\Lambda^{M}=\operatorname{diag}\left(2-2 \cos \frac{(m-1) \pi}{M}\right)_{m=1}^{M}$. Hence they are inverses of each other and diagonalize $A_{2}^{M}$.

We further define matrices

$$
C_{\mathrm{f}}=C_{\mathrm{f}}^{M} \otimes C_{\mathrm{f}}^{N}, \quad C_{\mathrm{b}}=C_{\mathrm{b}}^{M} \otimes C_{\mathrm{b}}^{N},
$$

which diagonalize $\boldsymbol{A}_{x x}, \boldsymbol{A}_{y y}$, and $\boldsymbol{A}_{\Delta^{2}}$ :

$$
\boldsymbol{C}_{\mathrm{f}} \boldsymbol{A}_{x x} \boldsymbol{C}_{\mathrm{b}}=\Lambda_{x x}, \quad C_{\mathrm{f}} \boldsymbol{A}_{y y} C_{\mathrm{b}}=\Lambda_{y y}, \quad C_{\mathrm{f}} \boldsymbol{A}_{\Delta^{2}} \boldsymbol{C}_{\mathrm{b}}=\Lambda_{\Delta^{2}},
$$

where the diagonal matrices are given by

$$
\boldsymbol{\Lambda}_{x x}=\frac{1}{\Delta x^{2}} \Lambda^{M} \otimes I d^{N}, \quad \boldsymbol{\Lambda}_{y y}=\frac{1}{\Delta y^{2}} I d^{M} \otimes \Lambda^{N}, \quad \boldsymbol{\Lambda}_{\Delta^{2}}=\left(\boldsymbol{\Lambda}_{x x}+\boldsymbol{\Lambda}_{y y}\right)^{2} .
$$

For a vector $w \in \mathbb{R}^{M N}$ we introduce its Fourier coordinates $\hat{w}$ by

$$
\hat{w}=\boldsymbol{C}_{\mathrm{f}} w \quad w=\boldsymbol{C}_{\mathrm{b}} \hat{w} .
$$

We note that $\mathbf{1}^{T} w=0$ if and only if the first component of $\hat{w}$ is zero.

Most of the computations involved in the variational methods described in Sec. 1.2 can be done in the Fourier coordinates. The only time one needs to go back to the original coordinates is when evaluating the brackets (2.5) and (2.6), because they are nonlinear and involve the discretized mixed derivative operator $\boldsymbol{A}_{x y}$.

2.5. Alternative discretization of $-\partial_{x y}$. The fast Fourier transform provides us with another discretization of the mixed derivative which is not biased to the left or right. In an analogy to (2.12) and (2.11) we define

$$
\boldsymbol{\Lambda}_{x y}=\frac{1}{\Delta x \Delta y} \sqrt{\Lambda^{M}} \otimes \sqrt{\Lambda^{N}}, \quad \tilde{\boldsymbol{A}}_{x y}=\boldsymbol{S} \boldsymbol{\Lambda}_{x y} \boldsymbol{C}_{\mathrm{f}},
$$

where $\boldsymbol{S}$ is the fast sine transform matrix

$$
\boldsymbol{S}=S^{M} \otimes S^{N}, \quad S^{M}=\frac{1}{\sqrt{2 M}}\left[\begin{array}{c|c}
0 & \left(2 \sin \frac{(2 i-1)(j-1) \pi}{2 M}\right)_{i=1, j=2}^{M, M} \\
\vdots &
\end{array}\right] .
$$

Property (2.9) also holds with $\boldsymbol{A}_{x y}$ replaced by $\tilde{\boldsymbol{A}}_{x y}$.

Remark 2.1. When discretizing the problem on the full domain $\Omega$ with boundary conditions (1.4), we need to use different matrices in the $x$ and $y$-directions. In the $x$-direction we use the matrices described above, in the $y$-direction to discretize the second derivatives, for example, we use

$$
A_{2}=\left[\begin{array}{rrrrr}
2 & -1 & & & -1 \\
-1 & 2 & -1 & & \\
& \ddots & \ddots & \ddots & \\
& & -1 & 2 & -1 \\
-1 & & & -1 & 2
\end{array}\right]
$$

In this direction the fast Fourier transform is used instead of the fast cosine/sine transform.

3. Newton's method. We use Newton's method in two different ways. The first is to improve the numerical approximations obtained by the variational numerical methods. Since these are sometimes slow to converge, it is often faster to stop such an algorithm early and use its result as an initial guess for Newton's method. The second use for Newton's method is as part of a numerical continuation algorithm (see Sec. 3.3). 
3.1. Newton's method for given load parameter $\lambda$. This method can be used to improve solutions obtained by the MPA. Let $\lambda \in(0,2)$ be given. We are solving

$$
\mathscr{G}(w, \phi)=\left[\begin{array}{c}
\mathscr{G}_{1} \\
\mathscr{G}_{2}
\end{array}\right]=\left[\begin{array}{l}
\boldsymbol{A}_{\Delta^{2}} w-\lambda \boldsymbol{A}_{x x} w+\boldsymbol{A}_{x x} \phi-2[w, \phi]_{1} \\
-\boldsymbol{A}_{\Delta^{2}} \phi+\boldsymbol{A}_{x x} w-[w, w]_{2}
\end{array}\right]=\left[\begin{array}{l}
\mathbf{0} \\
\mathbf{0}
\end{array}\right]
$$

for $w$ and $\phi$ with zero average using Newton's method. The matrix we need to invert is

$$
\mathscr{G}^{\prime}(w, \phi)=\left[\begin{array}{cc}
\frac{\partial \mathscr{G}_{1}}{\partial w} & \frac{\partial \mathscr{G}_{1}}{\partial \phi} \\
\frac{\partial \mathscr{G}_{2}}{\partial w} & \frac{\partial \mathscr{G}_{2}}{\partial \phi}
\end{array}\right]=\left[\begin{array}{ll}
\boldsymbol{A}_{\Delta^{2}}-\lambda \boldsymbol{A}_{x x}-2 \boldsymbol{B}_{1} & \boldsymbol{A}_{x x}-2 \boldsymbol{B}_{2} \\
\boldsymbol{A}_{x x}-2 \boldsymbol{B}_{2}^{T} & -\boldsymbol{A}_{\Delta^{2}}
\end{array}\right],
$$

where

$$
\begin{aligned}
\boldsymbol{B}_{1}=\frac{\partial}{\partial w}[w, \phi]_{1} & =\frac{1}{2} \boldsymbol{A}_{x x}(\operatorname{diag} \phi) \boldsymbol{A}_{y y}+\frac{1}{2} \boldsymbol{A}_{y y}(\operatorname{diag} \phi) \boldsymbol{A}_{x x}-\boldsymbol{A}_{x y}^{T}(\operatorname{diag} \phi) \boldsymbol{A}_{x y} \\
\boldsymbol{B}_{2}=\frac{\partial}{\partial \phi}[w, \phi]_{1} & =\frac{1}{2}\left(\frac{\partial}{\partial w}[w, w]_{2}\right)^{T} \\
& =\frac{1}{2} \boldsymbol{A}_{x x}\left(\operatorname{diag} \boldsymbol{A}_{y y} w\right)+\frac{1}{2} \boldsymbol{A}_{y y}\left(\operatorname{diag} \boldsymbol{A}_{x x} w\right)-\boldsymbol{A}_{x y}^{T}\left(\operatorname{diag} \boldsymbol{A}_{x y} w\right) .
\end{aligned}
$$

From the properties of $\boldsymbol{A}_{x x}, \boldsymbol{A}_{y y}, \boldsymbol{A}_{x y}, \boldsymbol{A}_{\Delta^{2}}$ it follows that matrix $\mathscr{G}^{\prime}(w, \phi)$ is symmetric and singular, its nullspace is spanned by $\left[\begin{array}{l}\mathbf{1} \\ \mathbf{0}\end{array}\right],\left[\begin{array}{l}\mathbf{0} \\ \mathbf{1}\end{array}\right]$.

To describe how to find the inverse of $\mathscr{G}^{\prime}(w, \phi)$ on a subspace orthogonal to its nullspace, we introduce a new notation for the four blocks of $\mathscr{G}^{\prime}(w, \phi)$ from (3.2):

$$
\mathscr{G}^{\prime}(w, \phi)=\left[\begin{array}{ll}
\boldsymbol{G}_{11} & \boldsymbol{G}_{12} \\
\boldsymbol{G}_{12}^{T} & \boldsymbol{G}_{22}
\end{array}\right]
$$

For given vectors $u, \eta$ with zero average we need to find vectors $v, \zeta$ with zero average such that

$$
\left[\begin{array}{ll}
\boldsymbol{G}_{11} & \boldsymbol{G}_{12} \\
\boldsymbol{G}_{12}^{T} & \boldsymbol{G}_{22}
\end{array}\right]\left[\begin{array}{l}
v \\
\zeta
\end{array}\right]=\left[\begin{array}{l}
u \\
\eta
\end{array}\right]
$$

Let the tilde denote the block of the first $M N-1$ rows and columns of a matrix and $M N-1$ components of a vector. The matrix $\left[\begin{array}{cc}\tilde{\boldsymbol{G}}_{11} & \tilde{\boldsymbol{G}}_{12} \\ \tilde{\boldsymbol{G}}_{12}^{T} & \tilde{\boldsymbol{G}}_{22}\end{array}\right]$ is symmetric, nonsingular, and sparse. It can be inverted by a linear sparse solver. System (3.3) is then solved in the following steps:

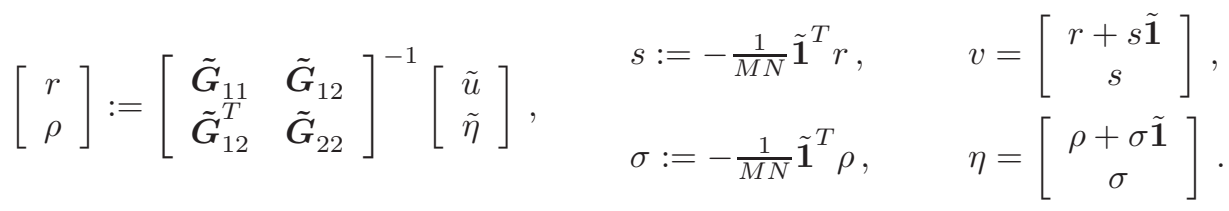

3.2. Newton's method for given $S$. This method can be used to improve solutions obtained by the CSDM and the CMPA. Let $C>0$ be given. We are looking for numerical solutions of (1.1-1.2) in the set $\mathscr{M}$ defined by (1.8). Hence we are solving

$$
\left[\begin{array}{l}
\boldsymbol{A}_{\Delta^{2}} w-\lambda \boldsymbol{A}_{x x} w+\boldsymbol{A}_{x x} \phi-2[w, \phi]_{1} \\
-\boldsymbol{A}_{\Delta^{2}} \phi+\boldsymbol{A}_{x x} w-[w, w]_{2} \\
-\frac{1}{2} w^{T} \boldsymbol{A}_{x x} w+C /(4 \Delta x \Delta y)
\end{array}\right]=\left[\begin{array}{l}
\mathbf{0} \\
\mathbf{0} \\
0
\end{array}\right]
$$

for $w$ and $\phi$ with zero average and $\lambda$ using Newton's method. The approach is very similar to that described in the previous section, the resulting matrix is symmetric, has just one more row and column than the matrix in (3.2). 
3.3. Continuation. To follow branches of solutions $(\lambda, w)$ of $(3.1)$ we adopt a continuation method described in [8]. We introduce a parameter $s \in \mathbb{R}$ by adding a constraint-pseudoarclength normalization (in the $\left(\lambda,\|w\|_{X}\right)$-plane). For a given value of $s$ we are solving

$$
\overline{\mathscr{G}}(w, \phi, \lambda)=\left[\begin{array}{c}
\mathscr{G}_{1} \\
\mathscr{G}_{2} \\
\mathscr{G}_{3}
\end{array}\right]=\left[\begin{array}{l}
\boldsymbol{A}_{\Delta^{2}} w-\lambda \boldsymbol{A}_{x x} w+\boldsymbol{A}_{x x} \phi-2[w, \phi]_{1} \\
-\boldsymbol{A}_{\Delta^{2}} \phi+\boldsymbol{A}_{x x} w-[w, w]_{2} \\
\theta\left\langle\dot{w}_{0}, w-w_{0}\right\rangle_{X}+(1-\theta) \dot{\lambda}_{0}\left(\lambda-\lambda_{0}\right)-\left(s-s_{0}\right)
\end{array}\right]=\left[\begin{array}{l}
\mathbf{0} \\
\mathbf{0} \\
0
\end{array}\right]
$$

for $w, \phi$ with zero average, and the load $\lambda$, where the value of $\theta \in(0,1)$ is fixed (e.g., $\theta=$ $\left.\frac{1}{2}\right)$. We assume that we are given a value $s_{0}$, an initial point $\left(\lambda_{0}, w_{0}\right)$ on the branch, and an approximate direction $\left(\dot{\lambda}_{0}, \dot{w}_{0}\right)$ of the branch at this point (an approximation of the derivative $\left.\left.\frac{d}{d s}(\lambda(s), w(s))\right|_{s=s_{0}}\right)$.

System (3.5) is solved for a discrete set of values of $s$ in some interval $\left(s_{0}, s_{1}\right)$ by Newton's method. Then, a new initial point on the branch is defined by setting $w_{0}=w\left(s_{1}\right), \lambda_{0}=\lambda\left(s_{1}\right)$, $s_{0}=s_{1}$, a new direction $\left(\dot{\lambda}_{0}, \dot{w}_{0}\right)$ at this point is computed and the process is repeated. The matrix we need to invert in Newton's method is

$$
\overline{\mathscr{G}}^{\prime}(w, \phi, \lambda)=\left[\begin{array}{cc}
\mathscr{G}^{\prime}(w, \phi, \lambda) & g \\
h^{T} & d
\end{array}\right], \quad g=\left[\begin{array}{c}
-\boldsymbol{A}_{x x} w \\
\mathbf{0}
\end{array}\right], h=\left[\begin{array}{c}
4 \theta \boldsymbol{A}_{\langle,\rangle} \dot{w}_{0} \Delta x \Delta y \\
\mathbf{0}
\end{array}\right], d=(1-\theta) \dot{\lambda}_{0},
$$

where $\boldsymbol{A}_{\langle,\rangle}=\boldsymbol{A}_{\Delta^{2}}+\boldsymbol{A}_{x x} \boldsymbol{A}_{\Delta^{2}}^{-1} \boldsymbol{A}_{x x}$.

Solving a linear system with this matrix amounts to solving system (3.3) for two right-hand sides. For a given $u \in \mathbb{R}^{2 M N}$ with $\left[\mathbf{1}^{T} \mathbf{1}^{T}\right] u=0$ and a given $\eta \in \mathbb{R}$ we want to solve

$$
\left[\begin{array}{cc}
\mathscr{G}^{\prime}(w, \phi, \lambda) & g \\
h^{T} & d
\end{array}\right]\left[\begin{array}{l}
v \\
\zeta
\end{array}\right]=\left[\begin{array}{l}
u \\
\eta
\end{array}\right]
$$

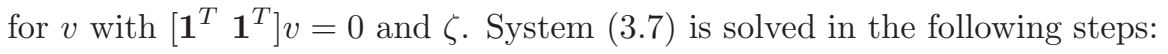

$$
\text { solve: } \begin{aligned}
\mathscr{G}^{\prime}(w, \phi, \lambda) v_{1}=g, & \zeta=\frac{\eta-h^{T} v_{2}}{d-h^{T} v_{1}}, \quad v=v_{2}-\zeta v_{1} .
\end{aligned}
$$

Remark 3.1. Note that in this implementation we simply follow a solution of the equation, there is no guarantee that this remains a local minimum, a MP-solution or constrained MP-solution (cf. Fig. 5.6).

Remark 3.2. Newton's method and continuation have been implemented only using a one-sided discretization of the mixed derivative $\partial_{x y}$ and only on the domain $\Omega_{\frac{1}{4}}$ assuming symmetry (1.9). The alternative discretization of $\partial_{x y}$ described in Sec. 2.5 uses the fast cosine/sine transform. The resulting matrix $\tilde{\boldsymbol{A}}_{x y}$ is not sparse and therefore we would obtain a dense block $\boldsymbol{G}_{12}$ in system (3.3) which would prevent us from using a sparse solver.

On the full domain $\Omega$ we assume periodicity of $w$ and $\phi$ in the $y$-direction. Hence for a discretization with a small step $\Delta y$ the matrix we invert when solving (3.3) would become close to singular. The shift in the $y$ direction is prevented by assuming the symmetry $w(x, y)=w(x,-y)$, $\phi(x, y)=\phi(x,-y)$.

4. Numerical solutions. We fix the size of the domain and the size of the space step for the following numerical computations: $a=b=100, \Delta x=\Delta y=0.5$. We obtain solutions using the variational techniques SDM, MPA, CSDM, and CMPA. Table 4.1 provides a summary of which discretization was used in which algorithm. 


\begin{tabular}{|l|c|c|c|c|}
\cline { 2 - 5 } \multicolumn{1}{c|}{} & \multicolumn{2}{c|}{ mixed derivative $\partial_{x y}$} & \multicolumn{2}{c|}{ computational domain } \\
\cline { 2 - 5 } \multicolumn{1}{c|}{} & one-sided & Fourier & full & $1 / 4$ \\
\hline variational methods & $\sqrt{ }$ & $\sqrt{ }$ & $\sqrt{ }$ & $\sqrt{ }$ \\
\hline Newton/continuation & $\sqrt{ }$ & & & $\sqrt{ }$ \\
\hline
\end{tabular}

TABLE 4.1

Summary of which spatial discretization was used in the different numerical methods.

4.1. A mountain-pass solution on the full domain $\Omega$. The first numerical experiments are done on the full domain $\Omega$, without symmetry restrictions, and with the unbiased (Fourier) discretization of $\partial_{x y}$ (Sec. 2.5). For a fixed load $\lambda=1.4$ we computed a mountain-pass solution using the MPA (Sec. 1.2.2). As end points were taken $w_{1}=0$ and a second point $w_{2}$ obtained by the SDM (here the initial point for the SDM was chosen to have a single peak centered at $x=y=0$ ). The graph of the solution $w_{\mathrm{MP}}$ is shown in Fig. 4.1 (left). The figure on the right shows $w_{\mathrm{MP}}$ rendered on a cylinder and we see it has the form of a single dimple. The value of shortening for this solution is $S\left(w_{\mathrm{MP}}\right)=14.93529$.

Alternatively, if we apply the CSDM with $S=14.93529$ and use a function with a single peak in the center of the domain as the initial condition $w_{0}$ we also obtain the same solution $w_{\mathrm{MP}}$, this time as a local minimizer of $E$ under constrained $S$.

We remark that although the MPA and the CSDM have a local character we have not found any numerical mountain-pass solution with the total potential $F_{\lambda}$ smaller than $F_{\lambda}\left(w_{\mathrm{MP}}\right)$ for $\lambda=1.4$. Similarly, using the CSDM we have not found any solution with energy $E$ smaller than $E\left(w_{\mathrm{MP}}\right)$ under the constraint $S=14.93529$. We briefly discuss the physical relevance of this solution in Sec. 6.

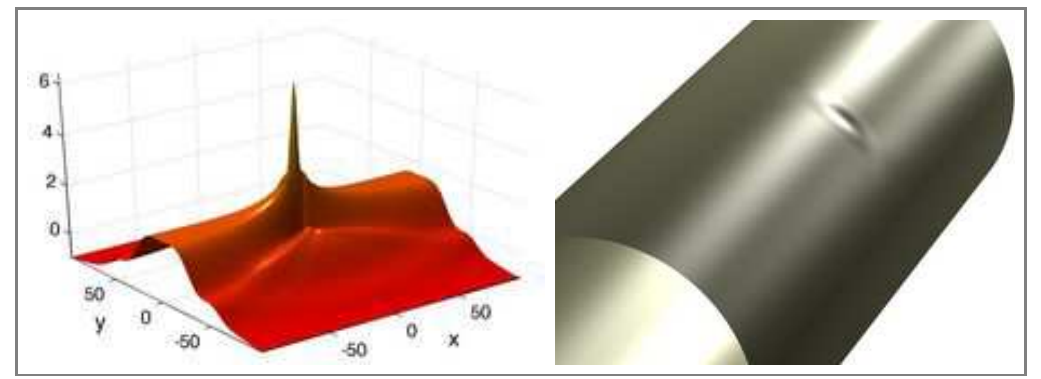

FiG. 4.1. Mountain-pass solution for $\lambda=1.4$ found using the MPA on the full domain $\Omega$ with $\partial_{x y}$ discretized using the fast Fourier transform.

4.2. Solutions under symmetry restrictions. The solution $w_{\mathrm{MP}}$ of Fig. 4.1 satisfies the symmetry property (1.9). In the computations described below we enforced this symmetry and worked on the quarter domain $\Omega_{\frac{1}{4}}$, thus reducing the complexity of the problem. In order to improve the variational methods by combining them with Newton's method we also discretized the mixed derivative $\partial_{x y}$ using left-sided finite differences. The influence of this choice on the numerical solution is described in Sec. 5.1.

4.2.1. Constrained steepest descent method. We first fixed $S=40$ and used the CSDM to obtain constrained local minimizers of $E$ described in Table 4.2. They are ordered according to the increasing value of stored energy $E$. Their graphs and renderings on a cylinder are shown in Fig. 4.2. Solution 1.1 is similar to the single dimple solution $w_{\mathrm{MP}}$ described above and according to Table 4.2 it has, indeed, the smallest value of $E$. 


\begin{tabular}{|c||c|c|c|c|c|}
\hline CSDM & $\lambda$ & $S$ & $E$ & $F_{\lambda}$ & same shape as MPA \\
\hline 1.1 & 1.108121 & 40 & 56.85636 & 12.53151 & 2.1 \\
\hline 1.2 & 1.299143 & 40 & 62.76150 & 10.79577 & 2.2 \\
\hline 1.3 & 1.316146 & 40 & 63.21646 & 10.57063 & 2.3 \\
\hline 1.4 & 1.311687 & 40 & 63.64083 & 11.17334 & 2.4 \\
\hline 1.5 & 1.309586 & 40 & 63.70623 & 11.32278 & 2.5 \\
\hline 1.6 & 1.328997 & 40 & 64.00875 & 10.84889 & 2.6 \\
\hline 1.7 & 1.344898 & 40 & 64.52244 & 10.72651 & 2.7 \\
\hline
\end{tabular}

TABLE 4.2

Numerical solutions obtained by the CSDM on $\Omega_{\frac{1}{4}}$ with $\partial_{x y}$ discretized using left-sided finite differences. Graphs are shown in Fig. 4.2. 

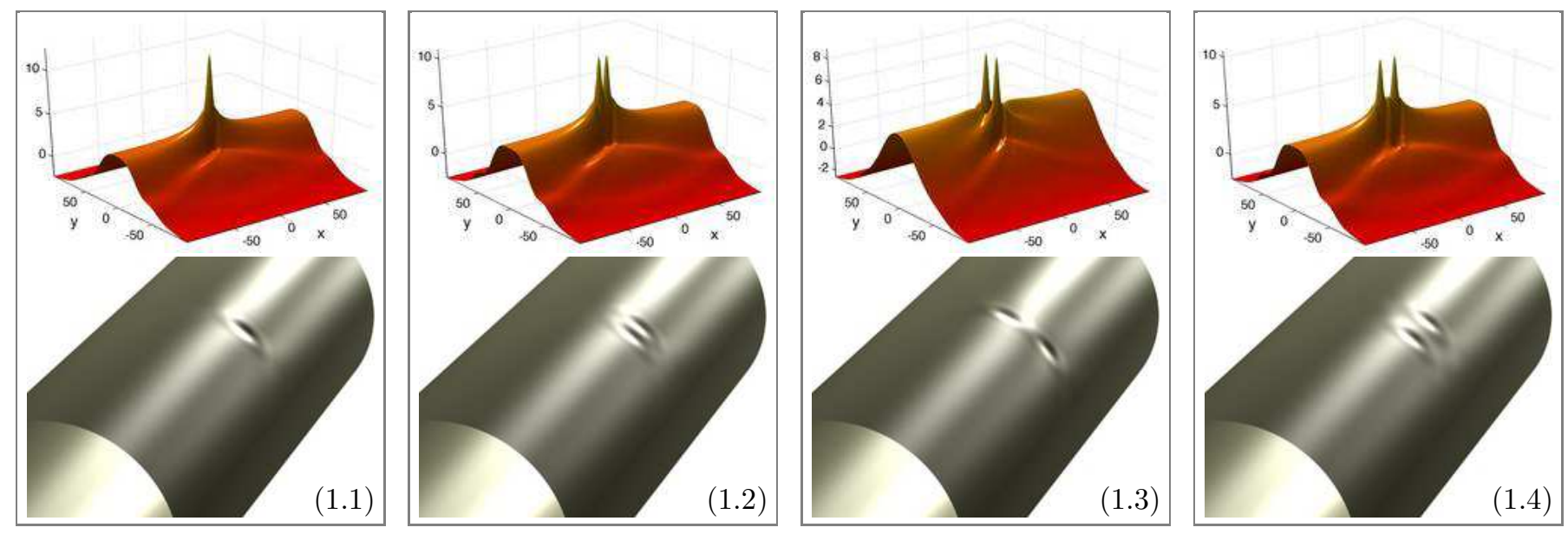

$\overleftrightarrow{\omega}$
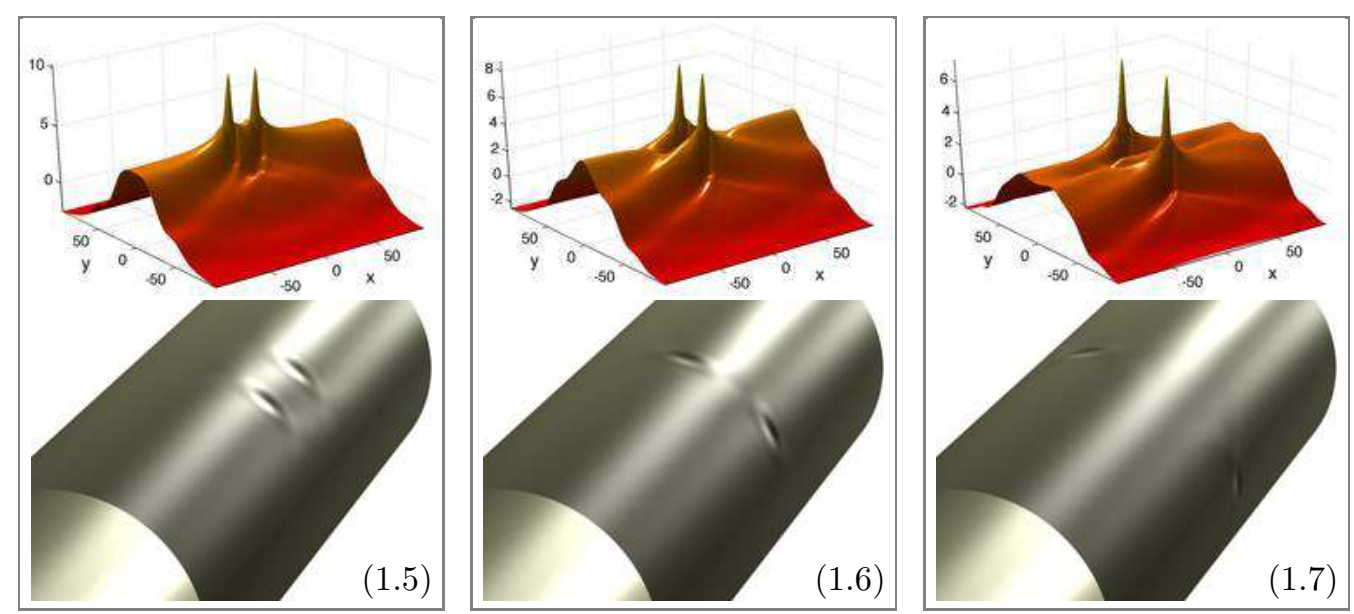

FIG. 4.2. Numerical solutions found using the CSDM with axial end shortening $S=40$. More details are in Table 4.2. 
4.2.2. Mountain-pass algorithm. We then used the MPA for fixed $\lambda=1.4$ and various choices of $w_{2}$ to obtain the local mountain-pass points of $F_{\lambda}$ described in Table 4.3. They are ordered according to the increasing value of the total potential $F_{\lambda}$. The shape of their graph is very similar to that of the CSDM solutions discussed above and depicted in Fig. 4.2 and we do not show their graphs here. Solution 2.1 is again the single dimple solution and the table shows that it has the smallest value of $F_{\lambda}$.

\begin{tabular}{|c||c|c|c|c|c|}
\hline MPA & $\lambda$ & $S$ & $E$ & $F_{\lambda}$ & same shape as CSDM \\
\hline 2.1 & 1.4 & 17.73822 & 29.42997 & 4.596460 & 1.1 \\
\hline 2.2 & 1.4 & 29.85121 & 49.08882 & 7.297132 & 1.2 \\
\hline 2.3 & 1.4 & 31.28849 & 51.39952 & 7.595635 & 1.3 \\
\hline 2.4 & 1.4 & 31.41723 & 52.01893 & 8.034809 & 1.4 \\
\hline 2.5 & 1.4 & 31.22992 & 51.84074 & 8.118852 & 1.5 \\
\hline 2.6 & 1.4 & 32.77491 & 54.15818 & 8.273314 & 1.6 \\
\hline 2.7 & 1.4 & 34.19888 & 56.56472 & 8.686284 & 1.7 \\
\hline
\end{tabular}

TABLE 4.3

Numerical solutions obtained by the MPA on $\Omega_{\frac{1}{4}}$ with $\partial_{x y}$ discretized using left-sided finite differences.

4.2.3. Constrained mountain-pass algorithm. We then fixed $S=40$ and applied the CMPA to obtain constrained local mountain passes of $E$ described in Table 4.4. They are again ordered according to the increasing value of stored energy $E$. Their graphs and renderings on a cylinder are shown in Figs. 4.3 and 4.4. As endpoints $w_{1}, w_{2}$ of the path in the CMPA we used the constrained local minimizers 1.1-1.7.

There are 21 possible pairs $\left(w_{1}, w_{2}\right)$ to be used but only 19 solutions in Table 4.4. The algorithm did not converge for the following three pairs: $(1.1,1.3),(1.5,1.6)$, and $(1.4,1.6)$, most likely due to the complicated nature of the energy landscape between these endpoints. On the other hand, two choices of pairs denoted by $*$ and $\dagger$ in the Table yielded two solutions each. When the path is deformed it sometimes comes close to another critical point of $E$ which is not a constrained mountain pass. In that case the algorithm slows down and one can apply Newton's method to such a point. It is a matter of luck whether Newton's method converges. The CMPA then runs further and might converge to another point, this time a constrained mountain-pass point. And finally, two choices of $\left(w_{1}, w_{2}\right)$ yielded the same solution 3.3. 


\begin{tabular}{|c||c|c|c|c|c|}
\hline CMPA & $\lambda$ & $S$ & $E$ & $F_{\lambda}$ & end points \\
\hline 3.1 & 1.310815 & 40 & 63.98996 & 11.55737 & $1.2,1.4$ \\
\hline 3.2 & 1.332112 & 40 & 64.38609 & 11.10161 & $1.3,1.6$ \\
\hline 3.3 & 1.447626 & 40 & 66.49032 & 8.585294 & $1.2,1.3$ or $1.3,1.4$ \\
\hline 3.4 & 1.440841 & 40 & 66.72079 & 9.087129 & $1.1,1.4$ \\
\hline 3.5 & 1.447594 & 40 & 66.97057 & 9.066810 & $1.1,1.6$ \\
\hline 3.6 & 1.484790 & 40 & 68.20637 & 8.814758 & $1.3,1.5$ \\
\hline 3.7 & 1.477769 & 40 & 68.23274 & 9.121955 & $1.1,1.5^{*}$ \\
\hline 3.8 & 1.482261 & 40 & 68.41086 & 9.120428 & $1.1,1.7$ \\
\hline 3.9 & 1.413917 & 40 & 68.56697 & 12.01028 & $1.1,1.2$ \\
\hline 3.10 & 1.520975 & 40 & 68.83818 & 7.999162 & $1.2,1.5$ \\
\hline 3.11 & 1.475705 & 40 & 69.00087 & 9.972652 & $1.1,1.5^{*}$ \\
\hline 3.12 & 1.532000 & 40 & 69.27379 & 7.993781 & $1.4,1.5^{\dagger}$ \\
\hline 3.13 & 1.527108 & 40 & 69.35834 & 8.274019 & $1.2,1.6$ \\
\hline 3.14 & 1.551762 & 40 & 69.47838 & 7.407904 & $1.4,1.5^{\dagger}$ \\
\hline 3.15 & 1.547955 & 40 & 69.68292 & 7.764712 & $1.6,1.7$ \\
\hline 3.16 & 1.539785 & 40 & 69.78487 & 8.193480 & $1.5,1.7$ \\
\hline 3.17 & 1.546480 & 40 & 69.85900 & 7.999795 & $1.3,1.7$ \\
\hline 3.18 & 1.549780 & 40 & 70.16253 & 8.171339 & $1.2,1.7$ \\
\hline 3.19 & 1.561117 & 40 & 70.74117 & 8.296474 & $1.4,1.7$ \\
\hline
\end{tabular}

TABLE 4.4

Numerical solutions obtained by the CMPA/Newton on $\Omega_{\frac{1}{4}}$ with $\partial_{x y}$ discretized using left-sided finite differences. Graphs are shown in Figs. 4.3, 4.4. 

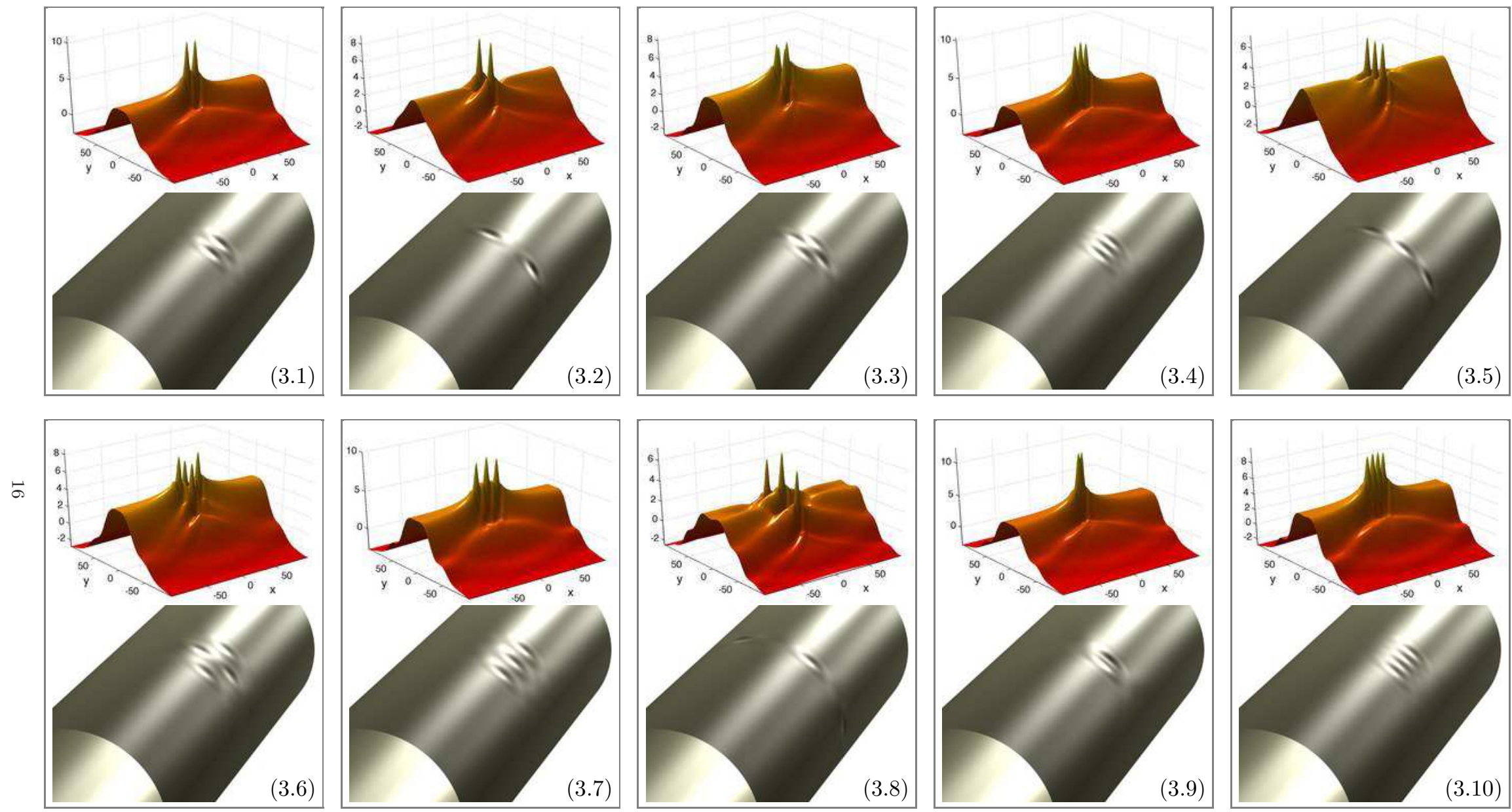

FIG. 4.3. Numerical solutions found using the CMPA/Newton with $S=40$. More details are in Table 4.4. 

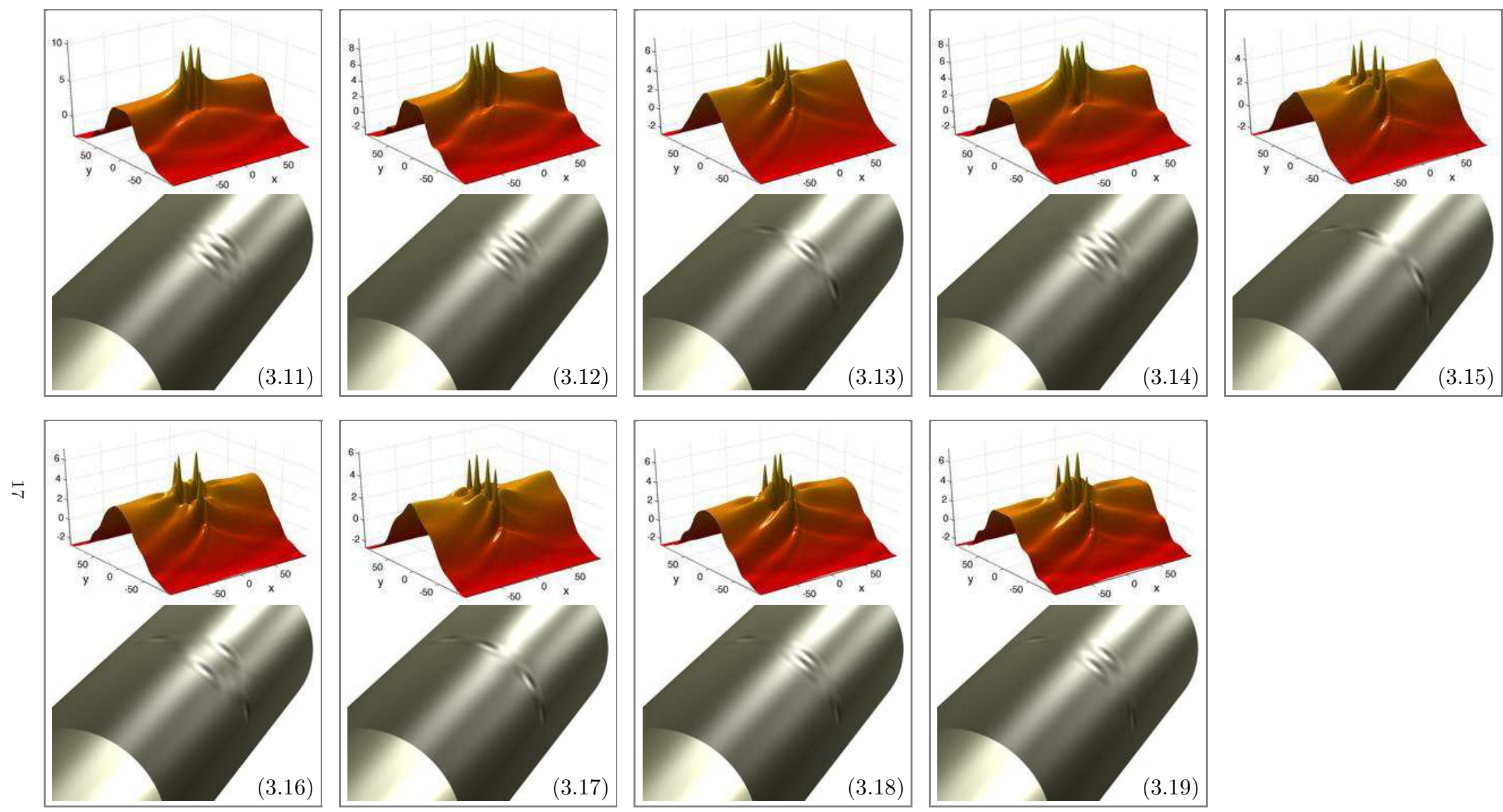

FIG. 4.4. Numerical solutions found using the CMPA/Newton with $S=40$. More details are in Table 4.4. 


\section{Remarks on the numerics.}

5.1. Bias in the discretization of $\partial_{x y}$. In this section we examine the influence of the discretization of the mixed derivative $\partial_{x y}$ on the numerical solution. We recall that the mixed derivative $\partial_{x y}$ can be discretized using left/right-sided finite differences $(2.3),(2.4)$ or using the fast Fourier transform (Section 2.5). For comparison we use the single-dimple solution on $\Omega=$ $(-100,100)^{2}$ at load $\lambda=1.4$ obtained by the MPA.

Let $\Delta x=\Delta y=0.5$. Table 5.1 gives a list of numerical experiments together with the values of shortening and energy. Figure 5.1 shows a profile of the numerical solutions in the circumferential direction at $x=0$.

\begin{tabular}{|c|c|c|c|c|c|c|}
\hline domain & discretization of $\partial_{x y}$ & $\lambda$ & $S$ & $E$ & $F_{\lambda}$ & Figure 5.1 \\
\hline$\Omega$ or $\Omega_{\frac{1}{4}}$ & Fourier & 1.4 & 14.93529 & 24.71825 & 3.808850 & - \\
\hline$\Omega$ & left/right-sided & 1.4 & 14.93617 & 24.70828 & 3.797636 & - \\
\hline$\Omega_{\frac{1}{4}}$ & left-sided & 1.4 & 17.73822 & 29.42997 & 4.596460 & $\cdots \cdots \cdots \cdots$ \\
\hline$\Omega_{\frac{1}{4}}$ & right-sided & 1.4 & 12.81205 & 21.16342 & 3.226549 & ----- \\
\hline
\end{tabular}

TABLE 5.1

Single-dimple numerical solution obtained by the MPA with and without the symmetry assumption (1.9) and with various kinds of discretization of $\partial_{x y}$.

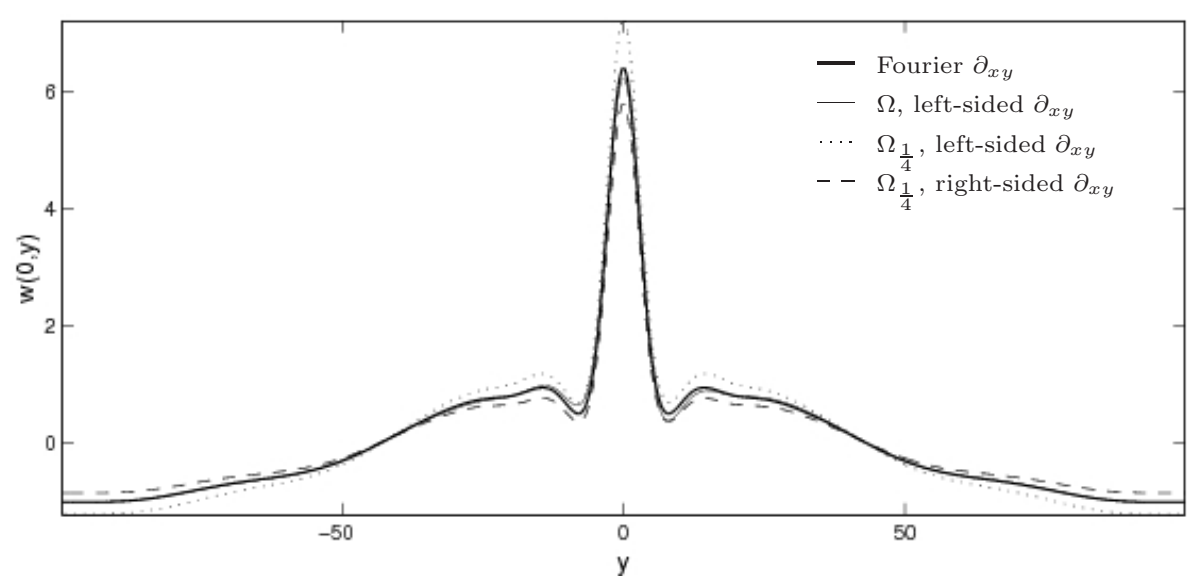

FIG. 5.1. Profile of the single-dimple numerical solution $w_{\mathrm{MP}}$ at $x=0$ obtained by the MPA with and without the symmetry assumption (1.9) and with various kinds of discretization of $\partial_{x y}$.

On the full domain $\Omega$ with no assumption on symmetry of solutions the discretization of $\partial_{x y}$ using the left/right-sided finite differences (2.3) provides a numerical solution that is slightly asymmetric (Fig. 5.1, thin solid line). The Fourier transform provides a symmetric solution (Fig. 5.1, thick solid line). The same numerical solution can be obtained on $\Omega_{\frac{1}{4}}$ under the symmetry assumption (1.9) with $\partial_{x y}$ discretized using the fast cosine/sine transform.

On $\Omega_{\frac{1}{4}}$ the symmetry of numerical solutions is guaranteed by assumption (1.9). The use of left/right-sided discretization of $\partial_{x y}$ does, however, have an influence on the shape of the numerical solution, as Fig. 5.1 shows (the dotted and the dashed line).

5.2. Convergence. We now turn to the influence of the size of the space step $\Delta x, \Delta y$ on the numerical solution. We run the MPA on $\Omega_{\frac{1}{4}}$ under the symmetry assumption (1.9) with $\partial_{x y}$ discretized using (a) the fast cosine/sine transform, (b) left-sided finite differences, (c) right-sided finite differences. We consider $\Delta x=\Delta y=0.5,0.4,0.3,0.2,0.1$, i.e., we take $200,250,333,500$, and 1000 points in both axis directions, respectively. Figure 5.2 illustrates convergence as $\Delta x, \Delta y \rightarrow 0$ of the numerical solutions obtained by various types of discretization of $\partial_{x y}$. 

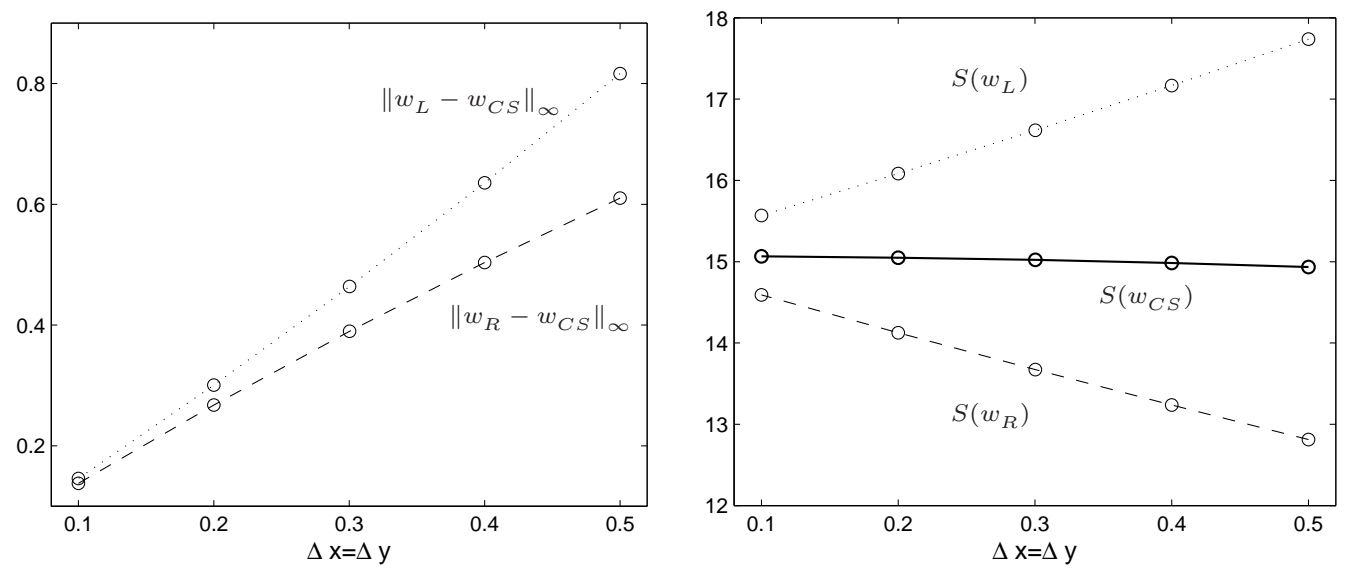

FIG. 5.2. Influence of the size of the space step $\Delta x, \Delta y$ on the numerical solution $w_{\mathrm{MP}}$ obtained by the MPA for three different kinds of discretization of $\partial_{x y}$. Let $w_{L}, w_{R}$ denote the numerical solutions obtained using the left and right-sided discretization of $\partial_{x y}$, respectively, $w_{C S}$ using the fast cosine/sine transform. Left: comparison of the solutions in the maximum norm; right: the value of shortening $S$.

5.3. Dependence on the size of the domain. As observed in [5], the localized nature of the solutions suggests that they should be independent of domain size, in the sense that for a sequence of domains of increasing size the solutions converge (for instance uniformly on compact subsets). Such a convergence would also imply convergence of the associated energy levels. Similarly, we would expect that the aspect ratio of the domain is of little importance in the limit of large domains.

We tested these hypotheses by computing the single-dimple solution on domains of different sizes and aspect ratios. In all the computations the space step $\Delta x=\Delta y=0.5$ is fixed. In order to use the continuation method of Sec. 3.3, we discretized $\partial_{x y}$ using the left-sided finite differences. We also assumed symmetry of solutions given by (1.9) and worked on $\Omega_{\frac{1}{4}}$. We recall the notation of computational domains, $\Omega=(-a, a) \times(-b, b), \Omega_{\frac{1}{4}}=(-a, 0) \times(-b, 0)$.

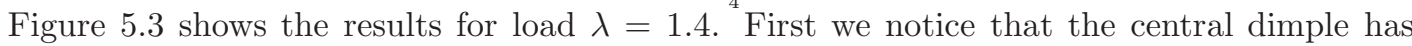
almost the same shape in all the shown cases. But there seems to be a difference in the slope of the "flat" part leading to this dimple. On domains with small $a$ (short cylinder) the derivative in the circumferential $y$-direction in this part is larger than on domains with larger $a$ (longer cylinder). The circumferential length $b$ seems to be less important for the shape of the solution: for example, the cases $(200,50)$ and $(200,100)$ look like restrictions of the case $(200,200)$ to smaller domains.

We take a closer look at domains of sizes $(a, b)=(100,100),(100,200),(200,100)$, and $(200,200)$ and the corresponding solutions $w_{100,100}, w_{100,200}, w_{200,100}$, and $w_{200,200}$ shown in the figure. We compare the first three with the last one, respectively. It does not make sense to compare the values of $w$ itself since the energy functional $F_{\lambda}$ depends on derivatives of $w$ only. We choose to compare $w_{x x}$ and $w_{y y}$. Table 5.2 gives the infinity norm of the relative differences. Fig. 5.4 shows graphs of the difference $w_{100,100}-w_{200,200}$ and of the second derivatives $\left(w_{200,200}-w_{100,100}\right)_{x x},\left(w_{200,200}-w_{100,100}\right)_{y y}$ on the subdomain $(-100,0)^{2}$.

We conclude that solutions on different domains compare well; the maximal difference in the second derivatives of $w$ is three orders of magnitude smaller than the supremum norm of the same derivative. We also observe that varying the length parameter $a$ while keeping the circumference parameter $b$ fixed causes larger changes in the numerical solution than varying the cylinder circumference while keeping the length fixed.

Another way of studying the influence of the domain size on the numerical solution is comparing solution branches obtained by continuation as described in Sec. 3.3. We start with the mountain-pass solution for $\lambda=1.4$ shown in Fig. 5.3 and continue it for both $\lambda>1.4$ and $\lambda<1.4$. The results are presented in Fig. 5.5. We observe that the branches corresponding to the con- 
$(a, b)=(50,50)$

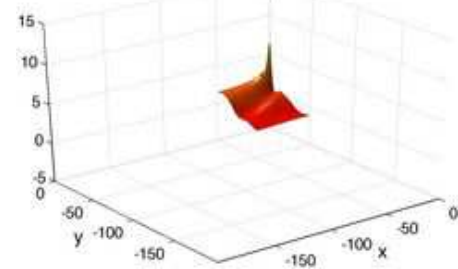

$(a, b)=(100,50)$

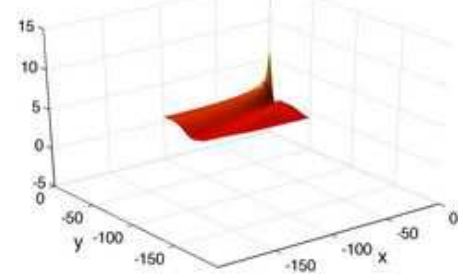

$(a, b)=(200,50)$

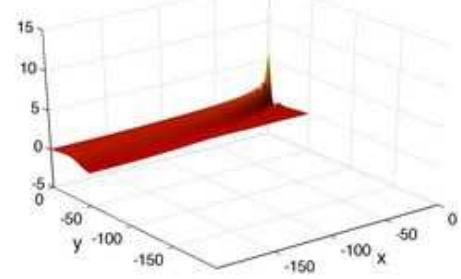

$(a, b)=(50,100)$

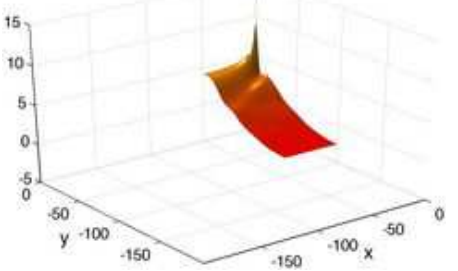

$(a, b)=(100,100)$

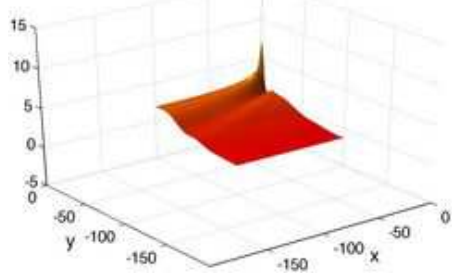

$(a, b)=(200,100)$

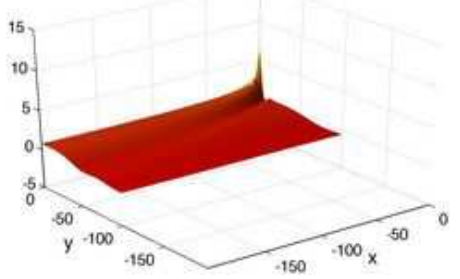

$(a, b)=(50,200)$

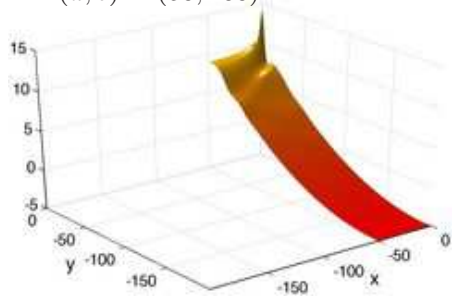

$(a, b)=(100,200)$

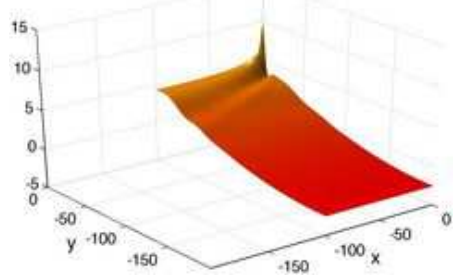

$(a, b)=(200,200)$

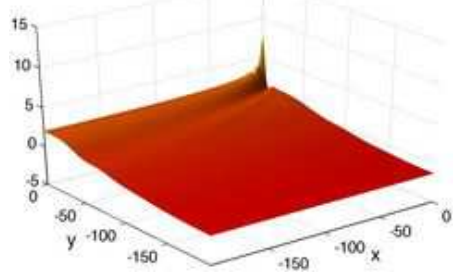

FIG. 5.3. The single-dimple mountain-pass solution with $\lambda=1.4$ computed under the assumption of symmetry (1.9) with left-sided discretization of $\partial_{x y}$ for various domain sizes.

$w_{100,100}-w_{200,200}$

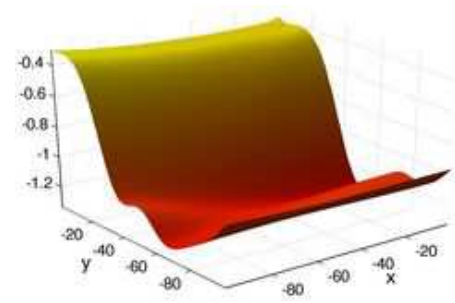

$\left(w_{200,200}-w_{100,100}\right)_{x x}$

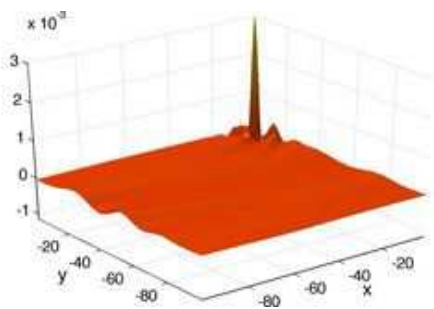

$\left(w_{200,200}-w_{100,100}\right)_{y y}$

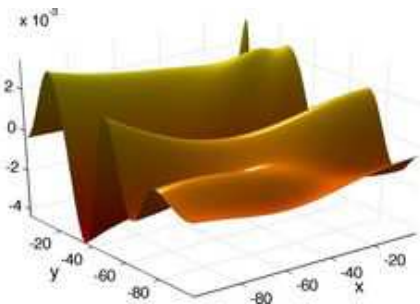

FIG. 5.4. Comparison of solutions $w_{100,100,} w_{200,200}$ from Fig. 5.3 obtained on square domains with $a=b=$ 100 and $a=b=200$, respectively, and their second derivatives. For a reference, we note that $\left\|\left(w_{200,200}\right)_{x x}\right\|_{\infty}=$ 1.064522 and $\left\|\left(w_{200,200}\right)_{y y}\right\|_{\infty}=0.8242491$.

\begin{tabular}{|l|c|c|}
\cline { 2 - 3 } \multicolumn{1}{c|}{} & $\frac{\left\|\left(w-w_{200,200}\right)_{x x}\right\|_{\infty}}{\left\|\left(w_{200,200}\right)_{x x}\right\|_{\infty}}$ & $\frac{\left\|\left(w-w_{200,200}\right)_{y y}\right\|_{\infty}}{\left\|\left(w_{200,200}\right)_{y y}\right\|_{\infty}}$ \\
\hline$w=w_{100,100}$ & $2.835 \cdot 10^{-3}$ & $5.313 \cdot 10^{-3}$ \\
\hline$w=w_{100,200}$ & $1.943 \cdot 10^{-3}$ & $4.917 \cdot 10^{-3}$ \\
\hline$w=w_{200,100}$ & $1.827 \cdot 10^{-4}$ & $9.638 \cdot 10^{-4}$ \\
\hline
\end{tabular}

TABLE 5.2

Comparison of the second derivatives of solutions from Fig. 5.3 computed on domains with $(a, b)=(100,100)$, $(100,200),(200,100)$, and $(200,200)$. 
sidered domains do not differ much for the range of $\lambda$ between approximately 0.71 and 2 . Below $\lambda \approx 0.71$ the size of the domain, particularly the length of the cylinder described by $a$, has a strong influence. The graph on the right shows that the larger (longer) the domain $\Omega$ the smaller the value of $\lambda$ at which the norm $\|w\|_{X}$ starts to rapidly increase for decreasing $\lambda$. The graph on the left shows the energy $F_{\lambda}(w)$ along a solution branch. The data shown here correspond to the ones in the graph on the right marked by a solid line. The dashed line in the right graph shows also some data after the first limit point is passed.
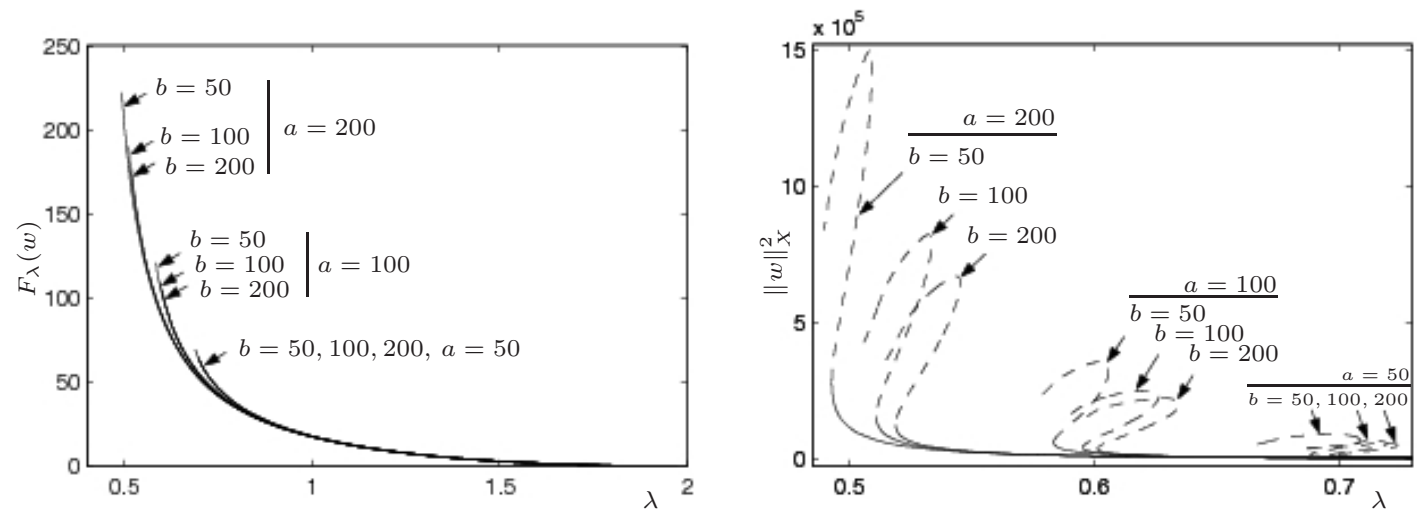

FIG. 5.5. Continuation of the single-dimple solution found as a numerical mountain pass for $\lambda=1.4$ on domains of various sizes for a range of values $\lambda$. Left: $F_{\lambda}(w)$ as a function of $\lambda$, right: $\|w\|_{X}$ as a function of $\lambda$.

Figure 5.6 shows how the graph of $w(x, y)$ changes as a solution branch is followed. Here we chose a square domain with $a=b=100$ and plotted the solution for four values of $\lambda$ (note that Figs. 5.6(c), 5.3(100,100), and 5.1(dotted line) show the same numerical solution). We observe that with decreasing $\lambda$ the height of the central dimple increases, the dimple becomes wider, and the ripples (present at $\lambda$ close to 2) disappear. In Fig. 5.6(a) we observe that new dimples are being formed next to the central dimple.

(a) $\lambda \approx 0.593$

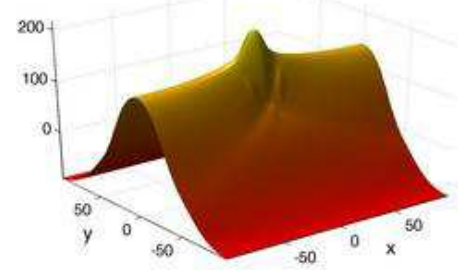

(b) $\lambda=0.61$ (mountain pass)
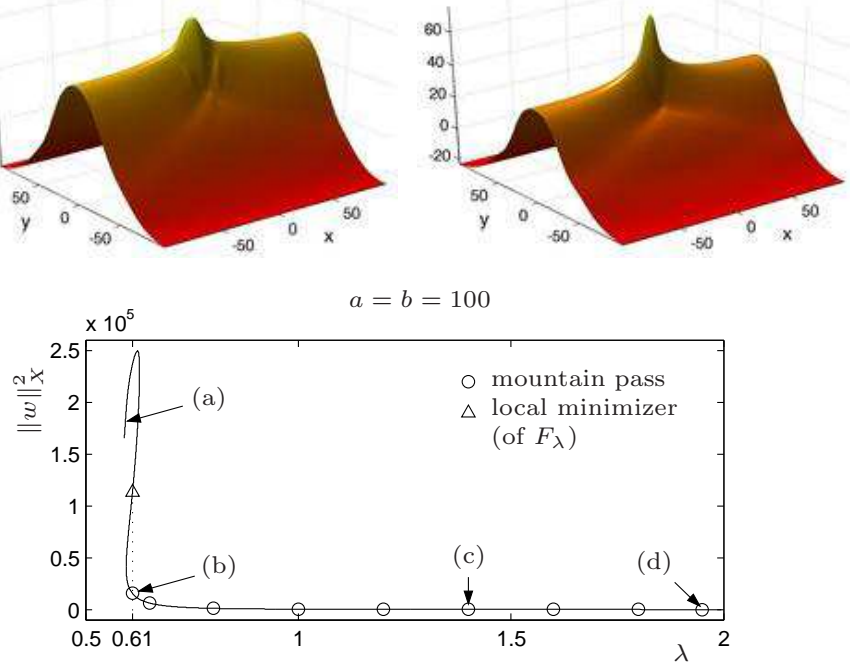

(c) $\lambda=1.4$ (mountain pass)

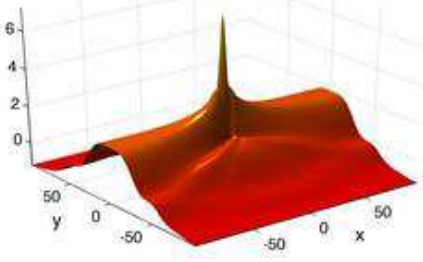

(d) $\lambda=1.95$ (mountain pass)

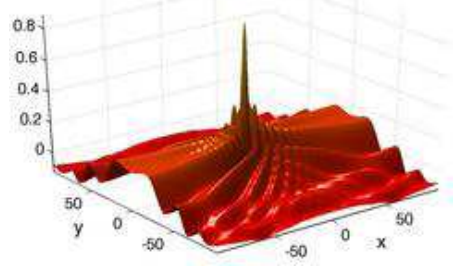

FIG. 5.6. A detailed look at the continuation of the single-dimple solution on the domain with $a=b=100$.

It should be remarked that although we started the continuation at $\lambda=1.4$ at a mountain-pass point, not all the points along a continuation branch are mountain passes. Since it is not feasible to use the MPA to verify this for each point, we chose just a few. Still on the example of the 
domain with $a=b=100$ in Fig. 5.6, the circles on the continuation branch mark those points that have also been found by the MPA (for $\lambda=0.61,0.65,0.8,1.0,1.2,1.4,1.6,1.8,1.95$ ). As described in Sec. 1.2.2, in order to start the MP-algorithm a point $w_{2}$ is needed such that $F_{\lambda}\left(w_{2}\right)<0$. The analysis in [5] shows that for a given $\lambda$ such a point exists provided the domain $\Omega$ is large enough and in practice it is found by the SDM of Sec. 1.2.1. This was, indeed, the case for all the chosen values of $\lambda$ except for $\lambda=0.61$. In this case, starting from some $w_{0}$ with a large norm, the SDM provides a trajectory $w(t)$ such that $F_{\lambda}(w(t))>0$ for all $t>0$. In fact, the steepest descent method converges to a local minimizer $w_{\mathrm{M}}$ with $F_{\lambda}\left(w_{\mathrm{M}}\right) \approx 76.1$. This is hence no mountain pass but, nevertheless, lies on the same continuation branch and is marked by a triangle in the figure. Despite $F_{\lambda}\left(w_{\mathrm{M}}\right)>0$ we can still try to run the MPA with $w_{2}=w_{\mathrm{M}}$. It converges and yields $w_{\mathrm{MP}}$ with $F_{\lambda}\left(w_{\mathrm{MP}}\right) \approx 94.8$ (marked by a circle at $\lambda=0.61$ and shown in graph (b)).

The comparison of solutions computed on different domains and their respective energies suggests that for each $\lambda$ we are indeed dealing with a single, localized function defined on $\mathbb{R}^{2}$, of which our computed solutions are finite-domain adaptations. Based on this suggestion and the above discussion of the mountain-pass solutions we could, for example, conclude that the mountain-pass energy

$$
V(\lambda, \Omega):=\inf _{w_{2}}\left\{F_{\lambda}\left(w_{\mathrm{MP}}\left(\lambda, \Omega, w_{2}\right)\right): F_{\lambda}\left(w_{2}\right)<0\right\}
$$

is a finite-domain approximation of a function $V(\lambda)$, whose graph almost coincides with that of $V(\lambda, \Omega)$ for $\lambda$ not too small (cf. Fig. 5.5 left).

\section{Discussion.}

6.1. Variational numerical methods. We have seen that given a complex energy surface many solutions may be found using these variational techniques. For example, for a fixed end shortening of $S=40$, Fig. 4.2, Fig. 4.3 and Fig. 4.4 are all solutions. Which of these solutions is of greatest relevance depends on the question that is being asked.

In the context of the cylinder (and similar structural applications) the mountain-pass solution from the unbuckled state $\left(w_{1}=0\right)$ with minimal energy is of physical interest. Often the experimental buckling load may be at $20-30 \%$ of the linear prediction from a bifucation analysis (in our scaling this corresponds to $\lambda=2$ ). This uncertainty in the buckling load is a drawback for design and so "knockdown" factors have been introduced, based on experimental data. It was argued in [5] that the energy of the mountain-pass solution $w_{\mathrm{MP}}$ in fact provides a lower bound on the energy required to buckle the cylinder and so these solutions provide bounds on the (observed) buckling load of the cylinder.

This example illustrates an important aspect of the (constrained) mountain-pass algorithm: its explicit non-locality. The algorithm produces a saddle point which has an additional property: it is the separating point (and level) between the basins of attraction of the end points $w_{1}$ and $w_{2}$.

Another technique to investigate a complex energy surface is to perform a simulated annealing computation, essentially to solve the SDM (or the CSDM) problem with additive stochastic forcing. The aim in these techniques is often to find a global minimizer (if it exists) where there are a large number of local minimizers. Here by either the MPA or the CMPA we find the solution between two such minima and so get an estimate on the surplus energy needed to change between local minima.

6.2. Numerical issues. The numerical issues that we encountered are of two types. First there are the requirements that are related to the specific problem of the Von Kármán-Donnell equations, such as the discretization of the mixed derivative and the bracket, and the fact that the solutions are symmetric and highly localized.

For other difficulties it is less clear. For smaller values of $\lambda$ each of the variational methods converged remarkably slowly. Newton's method provides a way of improving the convergence, but the question is relevant whether this slow convergence is typical for a whole class of variational problems. It would be interesting to connect the rate of convergence of, for instance, the SDM to certain easily measurable features of the energy landscape. 


\section{REFERENCES}

[1] C. Canuto, M. Y. Hussaini, A. Quateroni, And T. A. Zang, Spectral methods in fluid dynamics, Springer, 1987.

[2] Y. S. Choi And P. J. McKenna, A mountain pass method for the numerical solution of semilinear elliptic problems, Nonlinear Anal., 20 (1993), pp. 417-437.

[3] G. Friesecke and J. B. McLeod, Dynamic stability of non-minimizing phase mixtures, Proc. Royal Soc. London A, (1997), pp. 2427-2436.

[4] J. HoRÁK, Constrained mountain pass algorithm for the numerical solution of semilinear elliptic problems, Numer. Math., 98 (2004), pp. 251-276.

[5] J. Horák, G. J. Lord, And M. A. Peletier, Cylinder buckling: the mountain pass as an organizing center. To appear in SIAM Journal on Applied Mathematics.

[6] J. Horák And P. J. MCKenna, Traveling waves in nonlinearly supported beams and plates, in Nonlinear equations: methods, models and applications (Bergamo, 2001), vol. 54 of Progr. Nonlinear Differential Equations Appl., Birkhäuser, Basel, 2003, pp. 197-215.

[7] J. Horák And W. Reichel, Analytical and numerical results for the Fuč ik spectrum of the Laplacian, J. Comput. Appl. Math., 161 (2003), pp. 313-338.

[8] H. B. Keller, Numerical solution of bifurcation and nonlinear eigenvalue problems, in Applications of bifurcation theory (Proc. Advanced Sem., Univ. Wisconsin, Madison, Wis., 1976), Academic Press, New York, 1977, pp. 359-384. Publ. Math. Res. Center, No. 38.

[9] V. Maz'ya, S. Nazarov, and B. PlamenevskiJ, Asymptotic theory of elliptic boundary value problems in singularly perturbed domains. Vol. I, vol. 111 of Operator Theory: Advances and Applications, Birkhäuser Verlag, Basel, 2000. Translated from the German by Georg Heinig and Christian Posthoff.

[10] R. D. Richtmeyer and K. W. Morton, Difference Methods for Initial-Value Problems, Krieger, 2nd ed., 1994.

[11] G. Strang, Introduction to applied mathematics, Wellesley-Cambridge Press, Wellesley, MA, 1986.

[12] P. J. Swart And P. J. Holmes, Energy minimization and the formation of microstructure in dynamic anti-plane shear, Arch. Rat. Mech. Anal., 121 (1992), pp. 37-85. 